\title{
Chassis Coordinated Control for Full X-by-Wire Vehicles-A Review
}

\author{
Lei Zhang ${ }^{1,2}$ (D) Z Zhiqiang Zhang ${ }^{1,2}$, Zhenpo Wang ${ }^{1,2^{*}}$, Junjun Deng ${ }^{1,2}$ and David G. Dorrell ${ }^{3}$
}

\begin{abstract}
An X-by-wire chassis can improve the kinematic characteristics of human-vehicle closed-loop system and thus active safety especially under emergency scenarios via enabling chassis coordinated control. This paper aims to provide a complete and systematic survey on chassis coordinated control methods for full X-by-wire vehicles, with the primary goal of summarizing recent reserch advancements and stimulating innovative thoughts. Driving condition identification including driver's operation intention, critical vehicle states and road adhesion condition and integrated control of X-by-wire chassis subsystems constitute the main framework of a chassis coordinated control scheme. Under steering and braking maneuvers, different driving condition identification methods are described in this paper. These are the trigger conditions and the basis for the implementation of chassis coordinated control. For the vehicles equipped with steering-by-wire, braking-by-wire and/or wire-controlled-suspension systems, state-of-the-art chassis coordinated control methods are reviewed including the coordination of any two or three chassis subsystems. Finally, the development trends are discussed.
\end{abstract}

Keywords: X-by-wire systems, Chassis coordinated control, Driving condition identification, Active safety

\section{Introduction}

Modern vehicles are being increasingly equipped with advanced sensors and electronic control systems to obtain full perception of the surroundings and provide effective driver assistance in complex and ever-varying driving scenarios. Perception, trajectory planning and motion control constitute the three major tasks of supervisory control systems in connected and autonomous vehicles (CAVs) [1]. These have the potential of reducing driver fatigue and improving transport safety and efficiency [2-5]. Advanced X-by-wire chassis, which can include drive-by-wire, steer-by-wire, brake-by-wire and active/semi-active suspension subsystems, is necessary for autonomous driving. Efficient and coordinated control of X-by-wire chassis subsystems holds the key to the performance and safety of CAVs.

\footnotetext{
${ }^{*}$ Correspondence: wangzhenpo@bit.edu.cn

${ }^{1}$ National Engineering Laboratory for Electric Vehicles, Beijing Institute

of Technology, Beijing 100081, China

Full list of author information is available at the end of the article
}

Several active control systems (ACSs) have been developed based on X-by-wire chassis subsystems, such as Active Front Steering (AFS) [6], Direct Yaw-Moment Control (DYC) [7], Electronic Stability Control (ESC) [8], Electronic-Hydraulic Braking System (EHB) [9] and Active Suspension System (ASS) [10]. By applying an additional steering angle to the front wheels, the exclusive implementation of an AFS can potentially improve vehicle lateral stability without explicitly influencing longitudinal vehicle velocity. However, due to the limitations of system characteristics and tire force saturation, the AFS has a marginalized effect either when the additional yaw moment demand is too large or when the vehicle runs at its stability boundaries. In contrast, DYC can generate a desirable additional yaw moment by applying differential driving or braking torques to both wheel sides. ESC is a kind of active safety system which expands the functions of Anti-lock Braking System (ABS) and Traction Control System (TCS). EHB enables the decoupling of the hydraulic and the regenerative braking, and has
Springer Open

(c) The Author(s) 2021. This article is licensed under a Creative Commons Attribution 4.0 International License, which permits use, sharing, adaptation, distribution and reproduction in any medium or format, as long as you give appropriate credit to the original author(s) and the source, provide a link to the Creative Commons licence, and indicate if changes were made. The images or other third party material in this article are included in the article's Creative Commons licence, unless indicated otherwise in a credit line to the material. If material is not included in the article's Creative Commons licence and your intended use is not permitted by statutory regulation or exceeds the permitted use, you will need to obtain permission directly from the copyright holder. To view a copy of this licence, visit http://creativeco mmons.org/licenses/by/4.0/. 
the potential of enhancing both longitudinal and lateral stabilities. Recently, the burgeoning development of electric vehicles (EVs) provides great opportunities for ACSs to be increasingly adopted in production vehicles [11-13]. In particular, in-wheel-motor-drive electric vehicles (IWMD EVs) employ four in-wheel motors installed inside each wheel hub for direct propulsion, and fast response and accurate control precision of these hub-motors contribute to efficient DYC implementation [14-18]. Generally, vehicle safety can be enhanced via efficient vehicle motion control during the implementation of an ACS. But each vehicle motion is subject to more than one ACS while each ACS can exert influence on several vehicle motions. The relationship between vehicle motions and conventional ACSs are succinctly illustrated in Figure 1.

Suspension systems aim to transmit road excitations and damp the vibration, which is strongly related to vehicle roll and lateral dynamics. However, due to the complexity of suspension modeling and the strong couplings of vertical dynamics and other vehicle motions, most chassis control methods ignore the influence of suspension. ASS can generate a vertical force at each wheel by changing the corresponding spring stiffness and damping parameters so as to control vehicle body attitude and thereby enhance the handling stability and driver comfort.

Multiple chassis control systems may share the same actuators, so conflicts and interferences can emerge because of different control objectives. Recent research shows that chassis coordinated control can take advantage of each chassis subsystem to improve vehicle stability, handling performance, and ride comfort through the coordinated control of longitudinal, lateral, and vertical forces, especially under critical circumstances. It is widely recognized that the complex chassis coordinated control task can be achieved through a three-layer hierarchical structure, which is illustrated in Figure 2, where:

(1) The upper supervisor layer is responsible for identifying current driving condition based on critical vehicle states and driver's inputs. Through various

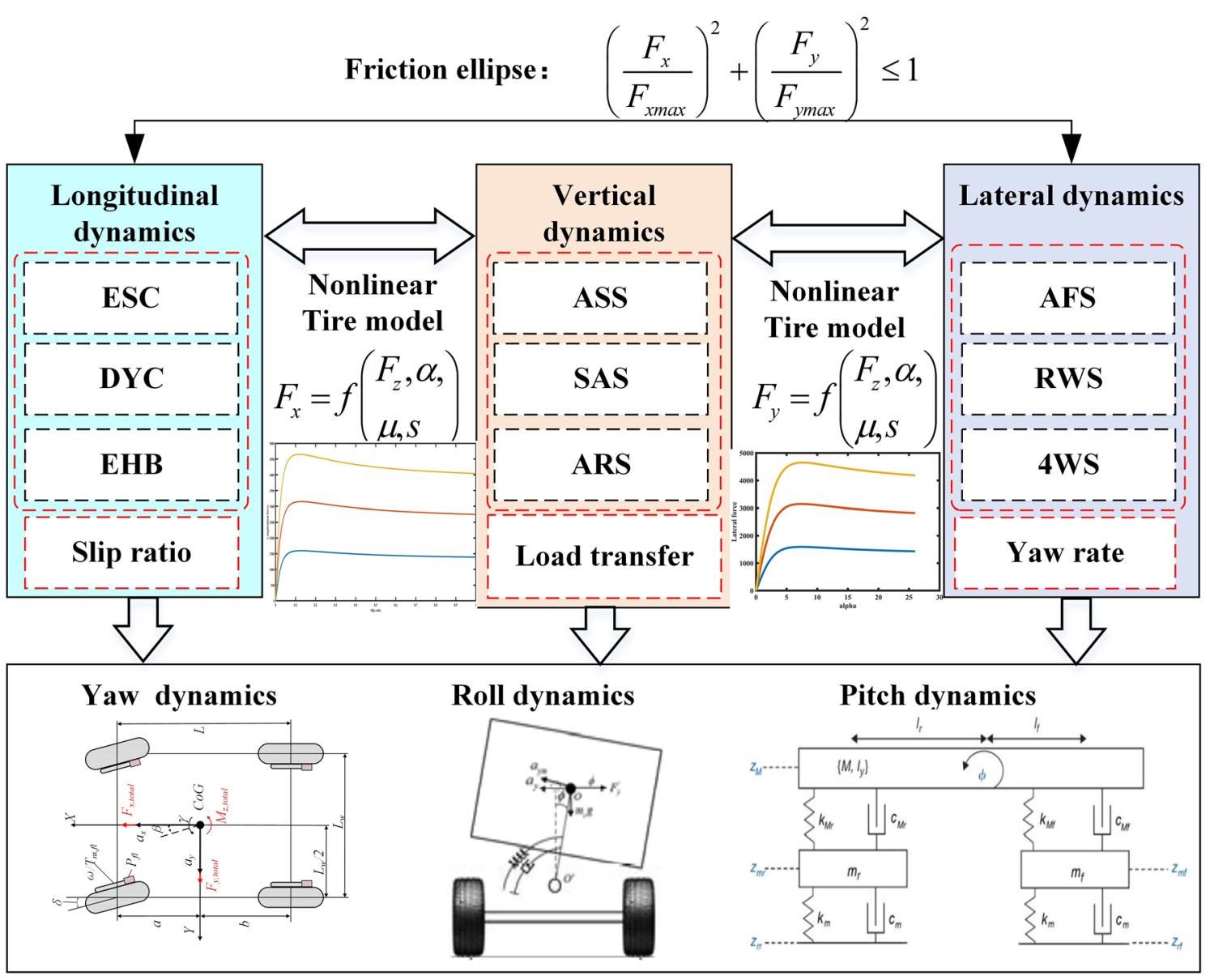

Figure 1 The relationship between vehicle motions and conventional ACSs 


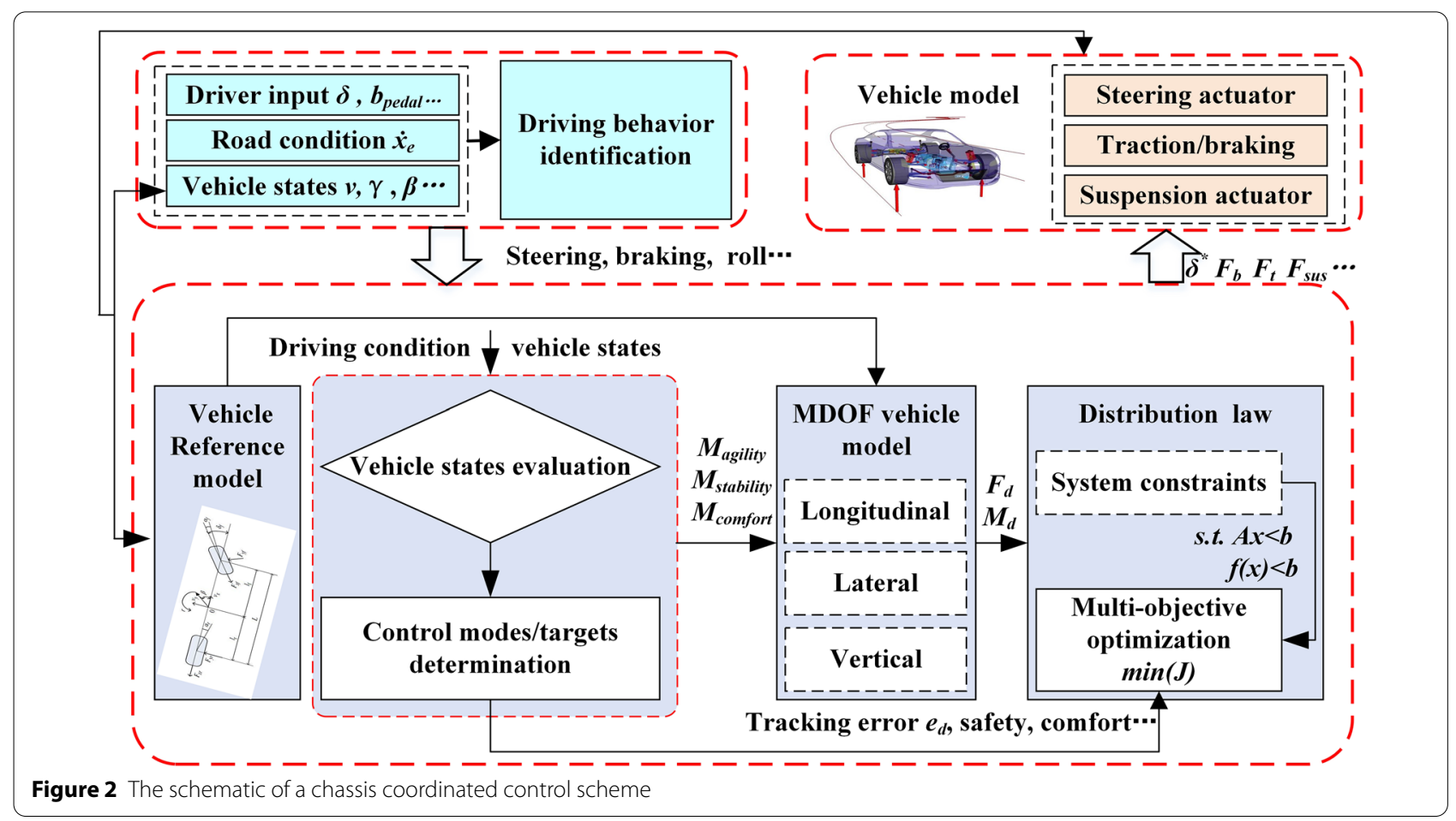

pattern recognition algorithms, the subject vehicle can clearly recognize a driving mode such as straight running, cornering, lane change or U-turn. This is in addition to vehicle operating conditions, i.e., rollover state, steering characteristics, and braking intensity. The driving condition identification results can be used to trigger different coordinated control modes, and are employed as the preconditions for selective involvements of specific chassis subsystems.

(2) The second coordination layer utilizes driver's inputs and real-time vehicle states to calculate the reference vehicle signals and then the desired forces/yaw moment based on a reference vehicle model. The driving condition identification results are analyzed to determine which chassis subsystems are to participate in the coordinated control and then their respective contributions to realize the desired generalized forces/yaw moment.

(3) The third control layer decomposes and dispatches the control tasks to individual chassis actuators. By considering the working areas of each chassis subsystem and actuators characteristics, the control layer aims to control the involved subsystems to track their target states by minizing the deviations of actual and target states through various control approaches.
With the continuous development of advanced perception sensors and Vehicle-to-Everything technologies, recent research has highlighted the intertwined relationships among chassis coordinated control, trajectory planning and expanding perception of the surroundings [19, 20]. Route planning and velocity planning constitute two important parts of trajectory planning [21, 22]. A safe and collision-free route is first derived considering obstacles and road boundaries, and then velocity planning designates speeds and accelerations to each waypoint considering the kinematic constraints and the route planning results. Vehicle states can be formulated as the critical inputs in the upper supervisor layer.

The rest of the paper is organized as follows. Section 2 describes the approaches for driving condition identification and discusses their respective advantages, disadvantages, and application ranges. Section 3 presents an extensive survey on state-of-the-art chassis coordinated control methods reported in the literture. These can be divided into two categories based on subsystem involvement patterns: 1) any two of the three chassis subsystems are involved; and 2) all the chassis subsystems participate. The overall concluding remarks are given in Section 4.

\section{Driving Condition Identification}

For a full X-by-wire vehicle to successfully perform different tasks under various driving scenarios, chassis coordinated control requires accurate vehicle driving condition 


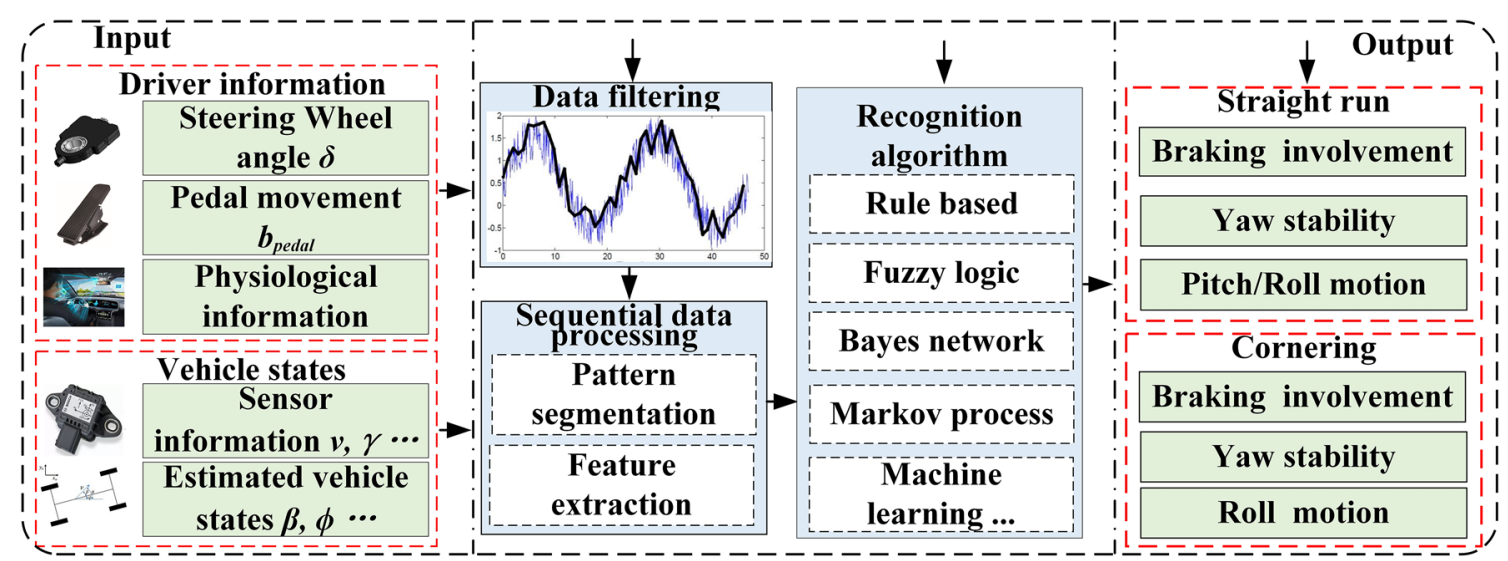

Figure 3 The flowchart of the driving condition recognition

identification. This includes vehicle states and road surface condition (e.g., road adhesion coefficient and road roughness) acquisition and driving behavior recognition. Critical vehicle states and road surface condition can be obtained directly by sensors such as gyroscopes and inertial navigation systems, or by using estimation algorithms [23-27]. It can be seen from Figure 3 that the detected vehicle states and road surface condition can be further used by the driving condition recognition module and classified into different operation conditions such as normal straight running or cornering and different working statuses such as stable and unstable. For each driving condition, the coordination layer would decide the main control objectives and involved chassis subsystems for chassis coordinated control. There has been much literature review on estimations of vehicle states [28-30], road adhesion coefficient [31] and road roughness [32]. Hence, this review merely focuses on driving behavior recognition.

\subsection{Steering Behavior Recognition}

Steering behavior recognition is responsible for detecting vehicle movement, speed and acceleration during cornering, which is essential for different ACSs to achieve effectual collision avoidance. For an autonomous vehicle cornering at high speeds, neither the steering response rate nor the overshoot can be satisfied in an ideal stabilization timeline without a timely steering behavior recognition. In other words, the timeliness and accuracy of steering behavior recognition can significantly influence the overall performance of chassis coordinated control. The literature shows that the existing steering behavior recognition methods can be sorted into several categories, the main ones being: rule-based [33], fuzzy logicbased [34], Hidden Markov Model-based (HMM-based) [35-38], neural network and machine learning-based [39-41], which are clearly shown in Table 1.
A rule-based steering behavior recognition method usually utilizes vehicle sensoring information related to vehicle yaw dynamics as inputs. In this regard, $\mathrm{Lu}$ et al. [42] proposed a steering behavior recognition algorithm based on steering wheel angle and its preset thresholds. Similarly, Choi et al. [33] presented a lane change maneuver recognition method using environmental and vehicle information as part of an emergency driving support system. Though rule-based methods have simple logic and consume little calculation resource, appropriate thresholds can be affected by car type, road surface condition and sensors' confidence [43]. Consequently, it is difficult to design a comprehensive set of rules to cover various steering behaviors, which leads to poor robustness and recognition accuracy. Hsiao et al. [34] developed a moving-vehicle behavior detection scheme based on a fuzzy inference system. This took the three-axis accelerometer information as inputs, resulting in a recognition rate of $80.31 \%$. However, the limitations of control accuracy and robustness lead to its applications only in early studies.

Recent studies indicate that steering behavior recognition is essentially a pattern recognition process and the HMM $[36,44]$ has been widely adopted in this area due to its strong ability in predicting event process states and dealing with time-series data [45]. The hidden relationship between vehicle states and the observed parameters is addressed as a general random process, which provides more correlations between current and previous states without exponentially increasing computational burden. Dejan [35] presented a basic and reliable HMM for recognizing current driving events. The proposed method treated vehicle velocities and accelerations in both longitudinal and lateral directions as inputs. It was found to be effective and accurate in recognizing the cornering and U-turn events. Pongsathorn et al. [36] developed a steering operation recognition-based DYC control algorithm 
Table 1 Steering behavior recognition

\begin{tabular}{|c|c|c|c|}
\hline $\begin{array}{l}\text { Reference } \\
\text { numbering }\end{array}$ & Algorithms & Inputs & Outputs \\
\hline [33] & Rule-based & $\delta, \dot{\delta}$ & Lane change intention \\
\hline [42] & Rule-based & $\delta, v, y, a_{x}, v_{c h}$ & $\begin{array}{l}\text { Straight run/cornering; } \\
\text { Stable/unstable }\end{array}$ \\
\hline [34] & Fuzzy-based & $a_{x^{\prime}} a_{y^{\prime}} a_{z}$ & $\begin{array}{l}\text { Straight run/cornering; } \\
\text { Accelerate/decelerate }\end{array}$ \\
\hline [36] & HMM-based & $\delta, \dot{\delta}$ & Lane keeping/lane change \\
\hline [44] & HMM-based & $\delta, \dot{\delta}$ & Normal driving/emergency driving \\
\hline [35] & HMM-based & $a_{x^{\prime}} a_{y^{\prime}} v$ & Emergency warning \\
\hline [37] & HMM-based & Lane position, $\delta, a$, braking intensity, $v, \gamma$ & $\begin{array}{l}\text { Lane keeping/lane change Accel- } \\
\text { erate/decelerate }\end{array}$ \\
\hline [38] & HMM-based & Lane position, $a_{x^{\prime}} a_{y^{\prime \prime}} v$ & $\begin{array}{l}\text { Straight run/cornering } \\
\text { Accelerate/decelerate }\end{array}$ \\
\hline [46] & R-HSMM-based & $\delta, a_{y}$ & Lane change intention \\
\hline [48] & Machine learning-based & $a_{x^{\prime}} a_{y^{\prime}} a_{z}$ & Straight run/cornering \\
\hline [49] & Machine learning-based & $\delta, v$ & Lane change \\
\hline [40] & Supervised learning-based & Lane position, $a_{x} a_{y}$ & Lane keeping/lane change \\
\hline [41] & Sequence learning-based & Signals, video images & Straight run/cornering \\
\hline$[51,56]$ & Machine learning-based & Smartphone navigation & Straight run/cornering \\
\hline [52] & Machine learning-based & Gaze accumulation and glance duration and frequency & Lane change intention \\
\hline [53] & Deep learning-based & Six-axis sensor & $\begin{array}{l}\text { Straight run/cornering } \\
\text { Normal/abnormal }\end{array}$ \\
\hline [54] & Deep learning-based & $a, v, \delta$ & $\begin{array}{l}\text { Straight run/cornering } \\
\text { Accelerate/decelerate }\end{array}$ \\
\hline [55] & Model-based & GNSS receiver, gyro, accelerometer and odometry. & $\begin{array}{l}\text { Straight running/cornering } \\
\text { Accelerate/decelerate }\end{array}$ \\
\hline [57] & HMM-based & Eye fixations, speed and $\delta$ & Lane change intention \\
\hline
\end{tabular}

and the overall scheme was verified as part of a drivervehicle system. An extended framework for lane change behavior recognition developed by Tran et al. [37] utilized a multi-dimensional feature vector including lane position, steering wheel angle, vehicle acceleration and brake pedal operation to recognize the steering intention based on an HMM. A K-means clustering method was used to convert the featured vector into discrete symbols. Similarly, Wu et al. [38] addressed a two-stage reasoningbased framework for ensuring driving safety. The first stage collected the recorded data containing lane bias, longitudinal and lateral accelerations, vehicle velocity and the distance to the front vehicle to train the HMM for various driving events including steering, lane change and normal driving. However, conventional HMMs often ignore the temporal features of driving data, which may lead to poor recognition accuracy at the beginning of steering behavior. The Recurrent Hidden Semi-Markov Model proposed in Ref. [46] provides a viable solution to handling the mentioned limitations.

Recent studies have also shown the potential of applying machine learning technologies to driving events recognition. Bayesian Networks (BNs) can provide suitable transition relationships between random signal variables and unknown vehicle states [47-49]. Hence, it is widely used in driving maneuver prediction. Veit et al. [39] proposed a maneuver prediction algorithm which considers the environmental situation and driver's behavior to predict lane change events based on BNs. However, it requires high-quality information of the subject vehicle, which means intensive computation requirement for data preprocessing. To release the computing burden of data processing, Mohammad et al. [40] introduced an integrated method to simultaneously realize model-based driving intention estimation and supervised learningbased motion prediction. The whole architecture could be summarized into a two-stage process: the motion intentions of traffic participants are first predicted using the game theory, and then the intention information is incorporated into a BNs-based classifier to identify current driving maneuvers.

Though BNs have shown the ability of dealing with classification problems, the feature value depends heavily on input information availability and information processing 


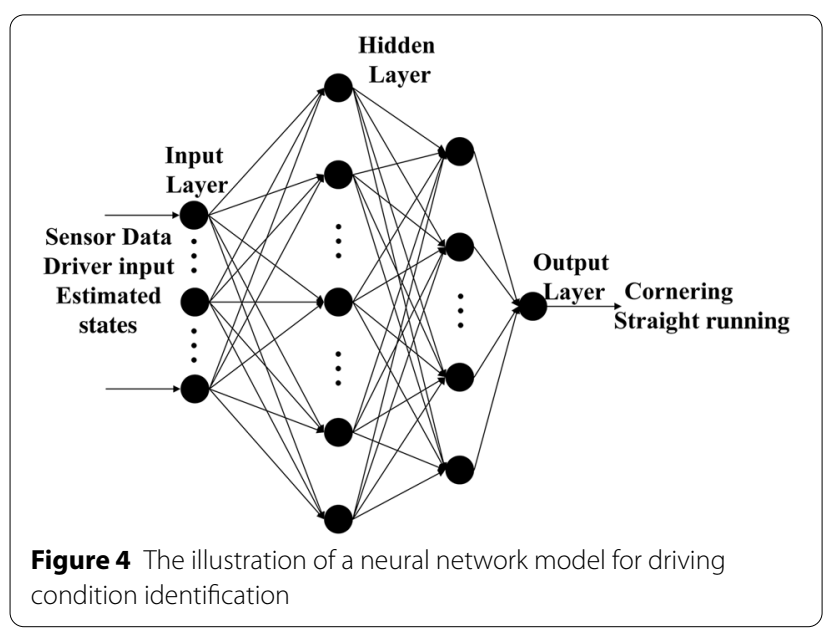

quality. Consequently, neural networks (NNs) have been commonly adopted because of use of independent probability model, good generalization capability and selflearning ability, which is shown in Figure 4. Han et al. [50] developed a driving intention recognition framework using NNs to predict a lane change maneuver. This contains a driver characteristic estimator and a driving behavior predictor. Further, Peng et al. [41] proposed a three-layer controller to predict steering and lane change behaviors based on a Long Short-Term Memory (LSTM) neural network. This uses vehicle control signals and video images as inputs.

The continuous penetration of advanced sensors and mobile units has solicited various studies focusing on combining these devices with maneuver recognition algorithms. For example, Ouyang et al. [51] proposed an ensemble learning-based model combined with a heuristic algorithm for smartphone-based steering behavior detection, where the algorithm collects the Global Positioning System (GPS), gyroscope and acceleration data from the smartphone. Advanced sensors, such as cameras, provide additional possibility for recognizing driving behaviors. Through a front camera that studies gaze dynamics, Martin et al. [52] addressed a machine-learning-based framework to classify driving behaviors using gaze accumulation and glance duration and frequency. It was shown that the proposed method could reach an accuracy of above $75 \%$ for lane changing prediction.

Conventional NNs are promising on driver behavior recognition, however, balancing the huge number of data samples required to obtain high accuracy and the limited computing resources remains a challenge. As a new research direction is the deep learning. This has made great advances in voice and image recognition because of its ability of enabling a virtual machine to imitate human sensing activities. Recently, driving behavior recognition based on the deep learning technology has also emerged. This has resolved the problems of small sample size and easy overfitting. For instance, Zhang et al. [53] proposed a driving behavior recognition method based on the deep learning technique. On-board sensor data is collected to extract the hidden features. A joint data argumentation scheme and a new multi-view convolutional $\mathrm{NN}$ model are developed for the training, learning and recognition of driving behaviors. Liu et al. [54] developed a visualization method called the driving color map based on the unsupervised learning. By mapping the extracted 3-dimentional hidden features to a red-green-blue color space, a driving color map was obtained. This was done by placing these colors in the corresponding positions on the map using observation sensors. The improved effectiveness of the proposed method was demonstrated under various traffic scenarios when compared to conventional principle component analysis methods.

Conventional driving states of ground vehicles can be represented by different kinematics models based on multiple model filters and pattern recognition approaches. Many studies of these can be found in the literature. For instance, Rafael et al. [55] addressed a bi-dimensional interactive multiple model based algorithm, which employed a reduced number of sensors inlcuding a Global Navigation Satellite System (GNSS) receiver, a gyro, an accelerometer and an odometry to predict driving behaviors. By considering sensor data fusion, interpretation and application, the proposed method was tested and proved to be effective in classifying longitudinal and lateral maneuvers.

During data collecting process, a fixed sliding window is often used. Since different steering modes may have disparate durations and intervals, it is difficult to find a suitable size and sliding steps. To release computational burden and improve recognition accuracy, Ouyang et al. [56] developed a Multi-Wave-Filter-based steering event recognition algorithm. A set of eight statistical sensor features reflecting major vehicle steering modes are identified by extracting the statistical features from different sensors. Machine learning methods are used to improve the training of classifiers. Lee et al. [57] proposed an early driver's intention prediction algorithm by modeling and analyzing driver's behaviors. This used structural pattern recognition based on context-free and context-sensitive grammars and leveraging eye fixation, vehicle speed and steering wheel angle as inputs. Through feature preprocessing, primitive selection and string pattern representation, the grammatical inference could be obtained. This can be used in the recognition module to classify cornering and lane changing maneuvers. 


\subsection{Braking Behavior/Roll State Recognition}

Braking is significant for safety and collision avoidance for ground vehicles. There are two main ways to improve vehicle safety by implementing braking operations. The first way is the emergency braking system, which uses advanced sensing devices such as Lidar, radar, cameras and lasers to detect obstacles and sends braking instructions to relevant actuators. Autonomous emergency braking systems, a typical emergency braking system, are now popular on private cars $[58,59]$. The second way is a braking assistance system that recognizes braking intention based on critical vehicle signals and driver's inputs before executing a braking maneuver. The first way is limited by sensors' performance, which is sensitive to the operating environments. Hence, recent work has focused on the braking assistance system, in which braking behavior recognition plays a vital role and provides opportunities to improve vehicle safety.

Conventional braking behavior recognition methods use behavioral data (e.g., the release speed of the accelerator pedal and the operation of the brake pedal) to detect braking behaviors. Katharina et al. [60] proposed a rule-based braking behavior recognition scheme using the acceleration and steering wheel angle of the vehicle and the movement and touch intensity of the brake pedal. An increasing number of vehicles are equipped with advanced sensors. On top of it, Frederik et al. [61] presented a rule-based braking intention recognition algorithm using the pedal operation and eye fixation of the driver collected by a front camera. A rule-based algorithm usually determines a braking maneuver based on the thresholds of related vehicle signals and the duration or frequency of each driving state; however, sensor uncertainty may bring unpredictable influence on the recognition results. To solve the problem, Li et al. [62] developed a driving style identification approach based on the transition patterns of each state. Though $\mathrm{NN}$ is widely accepted because of its ability to identify different types of driving behaviors from a large amount of data, the characteristic parameters in the training module can only be adjusted subjectively. The Gaussian mixture model (GMM) is a parameterization method for density estimation. It has the advantage of model adaptation and can generate the probability densities of arbitrary shapes. Hence, the GMM is often used in driving behavior and style identification. For example, Lv et al. [63] addressed a braking intensity recognition method based on the hybrid algorithm using supervised learning and the GMM. The GMM was used to cluster braking events automatically with brake pressure instead of simply setting thresholds for each vehicle state. Moreover, Wang et al. [64] combined the GMM and the HMM to predict the braking maneuvers of the proceeding vehicle, where the GMM was used to design driver modes and the HMM for predicting the trajectory. Similar research exists in Ref. [65].

Braking behaviors and intentions can be represented by driving operation data and the corresponding vehicle motion states, which is consistent with the HMM characteristics. A double-layer HMM was utilized to recognize acceleration and braking maneuvers in Ref. [66]. However, the proposed HMM showed high computational complexity when a longer time window was determined due to more hidden nodes in the model. The LSTM model is able to capture the long-range time-dependent relationship without incurring extra computational burden, which can make full use of driver's operation data to determine the connections between driving operations and the desired driving intentions. Jia et al. [67] proposed a LSTM-based neural network to detect abnormal emergency braking behaviors. Through classifying the abnormal acceleration point sets, the recognition rate reached 95\%. Wang et al. [68] presented a comparative study of the HMM and the LSTM to identify braking intentions. The results showed that the recognition accuracy of the LSTM was above 0.95, outperforming the HMM.

Observable behavioral actions are usually preceded by brain activities, so it is possible and reasonable to use human electroencephalography (EEG) signals to decode driver's braking behaviors. Teng et al. [69] developed an EEG-based emergency braking intention detecting algorithm. Their work analyzed the spectral features of emergency braking intentions of drivers and built a detection model for emergency braking intention recognition based on the regularization linear discriminant analysis. The experimental results showed that the proposed method could detect emergency braking intentions $420 \mathrm{~ms}$ after the onset of emergency situations with an accuracy of over $94 \%$.

Vehicle roll motion is highly sensitive to vehicle parameters, such as height of center of gravity (CG), wheelbase, and key states of vehicle. However, due to the complexity of roll dynamics and the coupling relationship between vehicle roll and longitudinal/lateral dynamics, current research often ignores the vehicle roll status during dynamics control, even in high-speed driving scenarios. Hence, there is an urgent need to monitoring the vehicle roll status to provide safety safeguards.

Unlike other critical vehicle state parameters, the indexes representing vehicle roll dynamics, e.g., roll angle and roll rate, are not easy to measure directly. In recent years, researchers have put forward a series of criteria to assess vehicle roll dynamics, which mainly include the static index-based [70-72], the Rollover Prevention Energy Reserve (RPER)-based [73], the Zero MomentPoint (ZMP)-based [74], the Load Transfer Ratio (LTR)based [75-78], and the Time-To-Rollover (TTR)-based 
[79], as well as several others. Therein, the most commonly-used can be addressed as

$$
L T R=\frac{F_{z o}-F_{z i}}{F_{z o}+F_{z i}},
$$

where $F_{z o}$ and $F_{z i}$ are the vertical loads of the outside and inside wheels.

Conventional static index-based methods usually take critical vehicle state parameters which are heavily related to roll dynamics, such as lateral acceleration and roll angle, as the roll stability judgement criteria [70, 71]. Therefore, the reliability of static index-based methods heavily relies on the high-precision estimation of vehicle roll angle and roll rate [80]. To this end, various methods have been developed to estimate critical vehicle states using the Kalman Filter and its variants as well as other methods [81-84]. The measured and estimated values of the critical representative vehicle states can then be compared with the predefined thresholds to determine the roll stability of the vehicle. However, they often ignore the uncertainties caused by vehicle type, driving scenario and road condition, and also barely describe the evolving trend of vehicle dynamics. Yin [73] investigated an RPER-based scheme to assess the real-time vehicle rollover risk by calculating the variation of the potential energy from the current state to the critical rollover state. This was on the basis of the coupling relationship between vehicle kinematics and dynamics effects and its subsystem parts. Lapapong et al. [74] developed a ZMPbased method to represent the rollover margin. By inferring the moment neutral point in the vertical direction, the vehicle showed strong roll stability if the ZMP was close to the wheelbase center. However, both the RPER and ZMP methods fail to reach a quantitative index so that it is hard to cover all the driving scenarios. Load transfer in rolling conditions can be addressed using the LTR. This was proposed in order to describe the tireroad attachment [76]. The LTR index is defined as the ratio of the differentce between the vertical forces acting on left- and right-side tires over their summation. This means its value is in the range from 0 to 1 . The rollover risk increases when the LTR value gets close to 1 . However, the actural vertical load of tires is difficult to obtain and needs to be observed using filtering algorithms [85]. Multi-algorithm-combination methods have recently been developed to improve the estimate precision in various vehicle operating conditions $[79,86]$.

\section{Coordinated Control}

Chassis coordinated control is often utilized to fulfill the requirements of vehicle active safety and fuel economy optimization based on driving condition identification. Drive/brake-by-wire (DBW/BBW), steer-by-wire (SBW), and suspension-by-wire are the major X-by-wire technologies. The involvement and control modes of these chassis subsystems are determined by classifying driving conditions. For example, the instability propensity under a sharp steering maneuver can be handled by either a combination of steering and braking subsystems or the coordinated control of steering, braking, and suspension subsystems. Generally, the chassis coordinated control can be divided into two categories according to subsystem participations: coordinated control of any two subsystems, or of all three subsystems.

\subsection{Driving/Braking and Steering Coordinated Control}

The vehicle planar dynamics consisting of longitudinal, lateral and yaw dynamics is illustrated in Figure 5, which is conventionally adopted in driving/braking and steering coordinated control. The body motion can be formulated as

$$
\begin{aligned}
& F_{x, t o t a l}=\left(F_{x, f l}+F_{x, f r}\right) \cos \delta+\left(F_{x, r l}+F_{x, r r}\right) \\
& -\left(F_{y, f l}+F_{y, f r}\right) \sin \delta-F_{w i n d},
\end{aligned}
$$

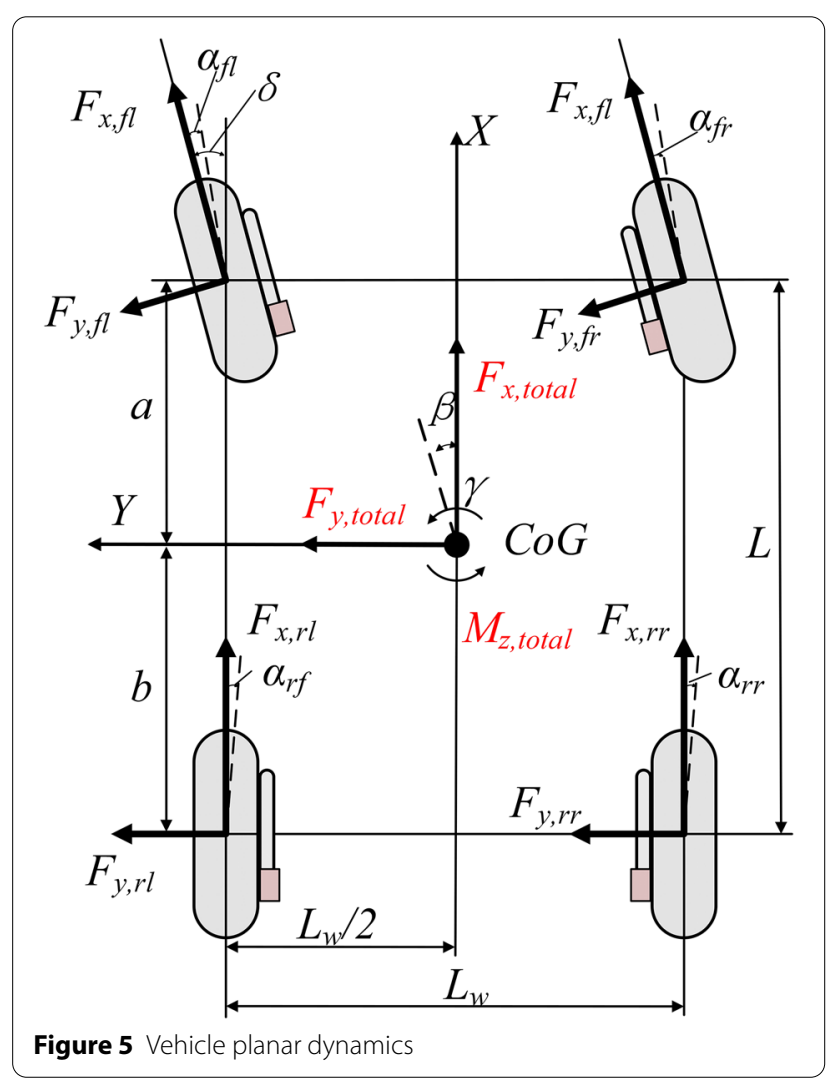




$$
\begin{aligned}
& F_{y, \text { total }}=\left(F_{x, f_{l}}+F_{x, f r}\right) \sin \delta+\left(F_{y, f l}+F_{y, f r}\right) \cos \delta \\
& +\left(F_{y, r l}+F_{y, r r}\right), \\
& M_{z, \text { total }}=\left(F_{x, f l}+F_{x, f r}\right) a \sin \delta \\
& +\left(F_{x, r r}+F_{x, f r} \cos \delta-F_{x, f l} \cos \delta-F_{x, r l}\right) \frac{L_{w}}{2} \\
& +\left(F_{y, f l}+F_{y, f r}\right) a \cos \delta-\left(F_{y, r l}+F_{y, r r}\right) b \\
& +\left(F_{y, f l} \sin \delta-F_{y, f r} \sin \delta\right) \frac{L_{w}}{2}
\end{aligned}
$$

where $F_{x, i j}$ and $F_{y, i j}(i j=f l, f r, r l, r r)$ stand for the longitudinal and lateral tire forces for each wheel, respectively; $F_{x, \text { total }}, F_{y, \text { total }}$ and $M_{\text {total }}$ are the total longitudinal force, lateral force and yaw moment, respectively; $\delta$ is the front wheel steering angle; $a$ and $b$ refer to the distances from the CG to the front and the rear axle, with $L=a+b ; F_{\text {wind }}$ is the wind resistance and $L_{w}$ is the wheelbase.

Moreover, as shown in Figure 6, the reference vehicle yaw rate $\gamma_{\text {ref }}$ and sideslip angle $\beta_{\text {ref }}$ can be obtained from a bicycle model, which can be given by

$$
\begin{aligned}
& \gamma_{r e f}=\min \left\{\left|\frac{v_{x}}{L\left(1+K v_{x}^{2}\right)} \delta\right|,\left|\gamma_{\max }\right|\right\} \operatorname{sgn}(\delta), \\
& \beta_{r e f}=0,
\end{aligned}
$$

where

$$
\begin{aligned}
& K=\frac{m}{L^{2}}\left(\frac{a}{k_{r}}-\frac{b}{k_{f}}\right), \\
& \gamma_{\max }=\frac{\mu g}{v_{x}},
\end{aligned}
$$

where $v_{x}$ is the longitudinal vehicle velocity and $\mu$ is the road adhesion coefficient; $k_{f}$ and $k_{r}$ are the cornering stiffness of the front and the rear axle, respectively; $m$ is the vehicle mass.

The operations on the driving/braking and steering systems expresses the driver's desired acceleration and yaw rate. These are the two frequently-manipulated subsystems in normal driving. Moreover, a widely recognized control scheme for the coordinated control of driving/braking and steering systems is shown in Figure 7. Optimizing vehicle agility, maneuverability, and handling stability by the combined control of steering and driving/braking systems has been studied extensively [87].

For the drive/brake-by-wire technology in IWMD EVs, the driving and the braking force can be easily measured and directly applied to each individual wheel. Moreover, the ratio between the front and the rear wheel braking force is variable, which is usually predefined as a constant in conventional vehicles. The flexible allocation of the driving and braking forces makes it achievable to improve vehicle stability, especially in some critical emergency scenarios. Recent years have witnessed the extensive applications of active safety systems such as Electronic Stability Program (ESP) [88] and DYC $[89,90]$. This confirms the potential for improving vehicle yaw dynamics by changing longitudinal tire forces through driving/braking and steering systems. For the steering system, SBW provides variable transmission ratio under different driving scenarios. Vehicles equipped with SBWs can achieve active steering, rear wheel steering (RWS), and even 4-wheel steering (4WS), without driver's intervention. The steering system can directly affect vehicle yaw dynamics by changing lateral tire forces. This is different from driving/braking systems that produce additional yaw moment by generating differential longitudinal tire forces. Much research has focused on the coordinated control of driving/braking and steering systems. As shown in Table 2, these common methods can be sorted into sliding model control (SMC)-based [91-93], model predictive control (MPC)-based [94-96], $H_{\infty}$ based [97], and linear quadratic regulator (LQR)-based $[98,99]$. There are also other methods in addition to these.

By providing an extra front steering angle corresponding to vehicle states, AFS can be treated as a transition from the Electric Power Steering (EPS) to the fully SBW. This can improve vehicle handling stability and driving comfort to some extent. However, due to the strong nonlinear characteristics of lateral

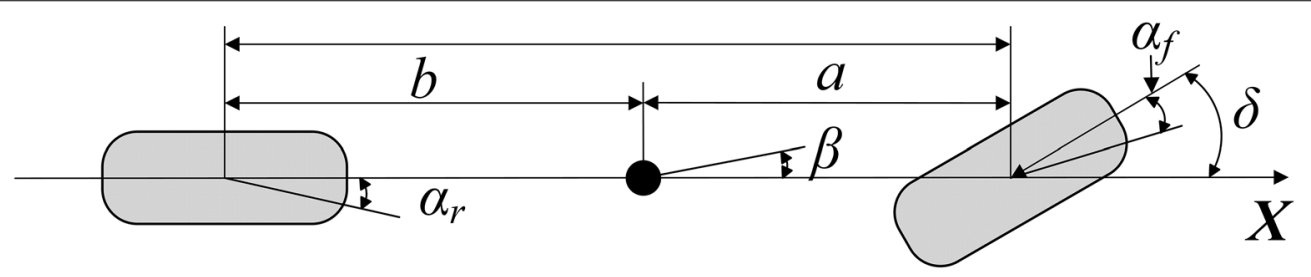

Figure 6 The reference 2-DOF vehicle model 


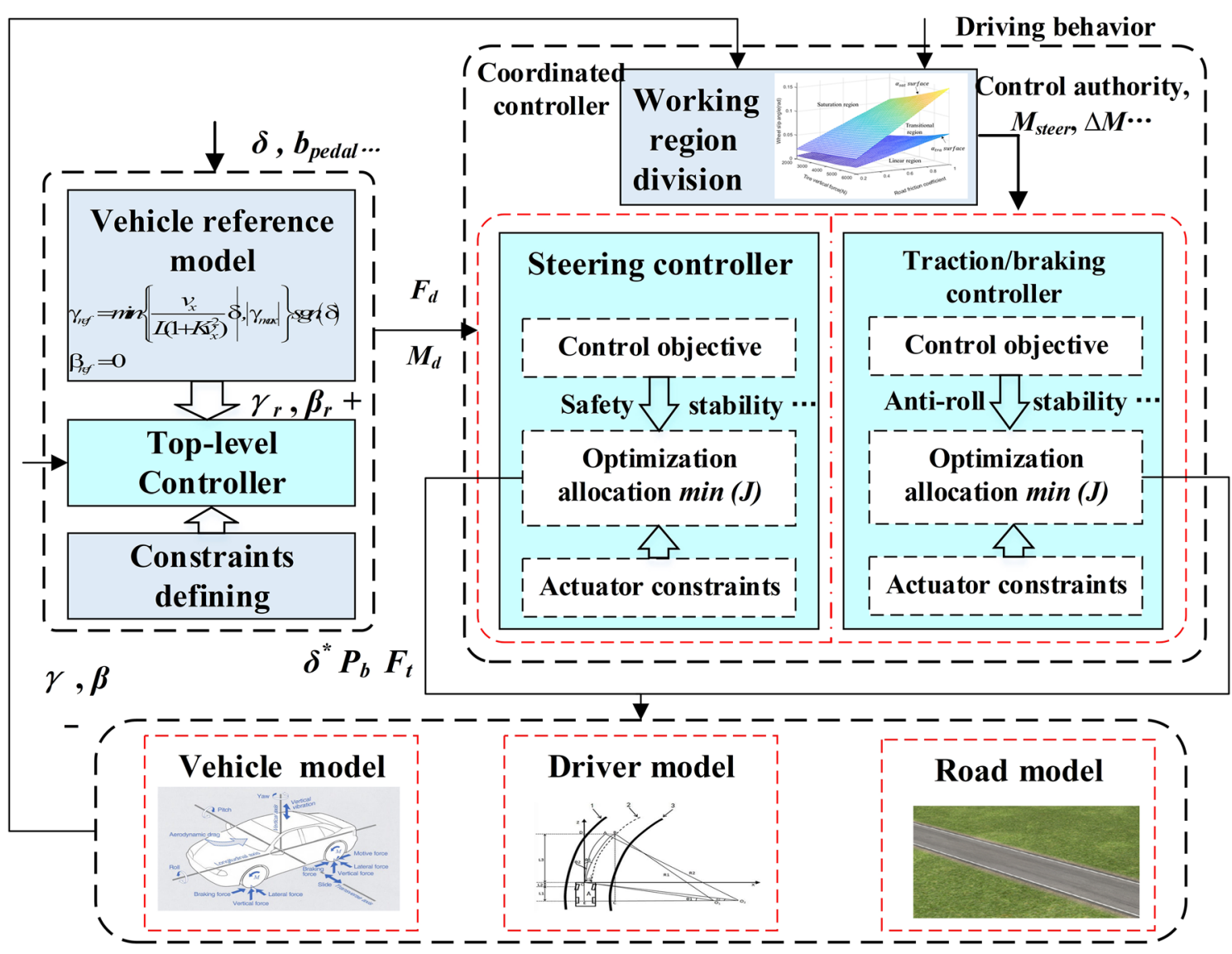

Figure 7 Coordinated control of driving/braking and steering systems

tire forces, AFS is only efficient when the vehicle is in a steady state. In other words, when the lateral acceleration and side slip angle of a vehicle increase and the vehicle enters the nonlinear region, it becomes challenging to obtain the desired yaw moment only from the AFS. Differential braking and DYC have demonstrated their effectiveness in enhancing vehicle lateral and yaw stability by applying braking or driving forces to individual wheels, especially when the vehicle operates in the nonlinear region with high driving speeds [100]. The principle of AFS and of DYC impacting the yaw moment can be represented as

$$
\Delta \delta_{f}=\frac{\Delta M_{A F S}}{a k_{f}},
$$

where $\Delta \delta_{f}$ is the additional front wheel steering angle and $\Delta M_{A F S}$ is the additional yaw moment generated by AFS.

$$
\left\{\begin{array}{l}
\left(F_{x, f l}+F_{x, f r}+F_{x, r l}+F_{x, r r}\right) \cdot r=T_{d}, \\
\frac{L_{w}}{2}\left(F_{x, f r}-F_{x, f l}\right) \cos \delta+\frac{L_{w}}{2}\left(F_{x, r r}-F_{x, r l}\right), \\
+a\left(F_{x, f l}+F_{x, f r}\right) \sin \delta=\Delta M,
\end{array}\right.
$$

where $T_{d}$ refers to the demand driving/braking torque and $\triangle M$ is the additional yaw moment generated by DYC.

Current research in coordinated control of AFS and differential braking/DYC aims to improve vehicle lateral and yaw stability while minimizing the interference on longitudinal vehicle velocity [101-103]. Stefano et al. [95] proposed an integrated MPC control method for AFS and differential braking to improve yaw stability while reducing the impact on longitudinal dynamics. A piecewise affine (PWA) approximation was applied to express the tire force characteristics and a tire slip angle envelop was utilized to describe the boundaries of the PWA region. The MPC algorithm took the braking 
Table 2 Coordination of braking and steering systems

\begin{tabular}{|c|c|c|c|c|}
\hline $\begin{array}{l}\text { Reference } \\
\text { numbering }\end{array}$ & Algorithms & Control inputs & Control target & Subsystems \\
\hline [87] & $\begin{array}{l}\text { Weighted pseudo-inverse based } \\
\text { control allocation }\end{array}$ & $M_{\text {yaw }}$ & $\begin{array}{l}\text { Maneuverability and lateral } \\
\text { stability }\end{array}$ & $\begin{array}{l}\text { ESC + AFS + 4-Wheel drive } \\
\quad(4 \mathrm{WD})\end{array}$ \\
\hline [88] & MPC & $M_{\text {yaw }}$ & $\begin{array}{l}\text { Directional stability and steer- } \\
\text { ability }\end{array}$ & $\mathrm{ESC}+$ active torque vectoring \\
\hline [90] & Model matching & $\beta_{\text {refi }} \gamma_{\text {ref }}$ & Lateral stability & RWS + DYC \\
\hline [100] & Constrained optimization & $M_{\text {yaw' }} F_{x}$ & $\begin{array}{l}\text { Agility, maneuverability, and } \\
\text { lateral Stability }\end{array}$ & $\mathrm{AFS}+\mathrm{ESC}$ \\
\hline [103] & Direct Lyapunov method & $V_{\text {refi }} Y_{\text {ref }}$ & Lateral stability, reduce $v$ error & AFS + DYC \\
\hline [95] & MPC & $a_{y^{\prime}} y_{\text {ref }}$ & $\begin{array}{l}\text { Reduce longitudinal speed error } \\
\text { and yaw stability }\end{array}$ & AFS + differential braking \\
\hline$[96,123,125]$ & MPC & $\beta_{\text {refi }} \gamma_{\text {ref }}$ & Lateral stability & AFS + differential braking \\
\hline [105] & Static-state feedback & $\beta_{\text {refi }} \gamma_{\text {ref }}$ & Handling stability & AFS + differential braking \\
\hline$[91,92]$ & SMC based & $\beta_{\text {refi }} \gamma_{\text {ref }}$ & Handling stability & $\mathrm{AFS}+\mathrm{DYC}$ \\
\hline [98] & Optimal guaranteed cost theory & $\beta_{\text {refi }} \gamma_{\text {ref }}$ & Handling stability & $\mathrm{AFS}+\mathrm{DYC}$ \\
\hline [106] & SMC & $F_{x d}, M_{\text {yaw }}$ & Path tracking performance & AFS + DYC \\
\hline [112] & SMC & $v_{x d !} v_{y d \prime} V_{\text {ref }}$ & Path tracking performance & Steer + brake \\
\hline [108] & Fuzzy $\mathrm{H}_{\infty}$ control & $\beta_{\text {refi }} \gamma_{\text {ref }}$ & Lateral stability & AFS + DYC \\
\hline [109] & Fuzzy & $M_{\text {yaw' }} F_{y}$ & Wheel slip & AFS + DYC \\
\hline$[116,117,120]$ & Constrained optimization & Desired force and yaw moment & Handling performance & $4 W S+4 W D$ \\
\hline [99] & LQR & & Fault tolerant & $4 W S+4 W D$ \\
\hline [126-129] & Nonlinear backstepping & $\begin{array}{l}\text { Path segmenta-tion, vehicle } \\
\text { states }\end{array}$ & Obstacle avoidance & Steer + brake \\
\hline$[72,77]$ & MPC, SMC & & Anti-roll & Steer + brake \\
\hline$[124,125]$ & MPC & & Regenerative braking & Steer + brake \\
\hline
\end{tabular}

forces of four wheels and the steering angle of the front wheels as the inputs while the yaw rate was selected as the observation variable. It is worth mentioning that a switched MPC was developed to reduce the computational burden. This assumed that the tire condition in the prediction horizon is unchangeable. However, ignoring the effects of vehicle side slip angle may lead to weak handling stability and low path tracking accuracy under high speed driving circumstances [104]. To solve this issue, Caglar et al. [105] developed a static-state feedback controller to ensure the robust performance against variable vehicle states such as vehicle speed, cornering state, braking stiffness and road adhesion condition. The nonlinear two-track yaw-plane vehicle model extended with wheel dynamics was used in the algorithm to track the desired vehicle side slip angle and yaw rate. The effectiveness of the proposed method was demonstrated in the fishhook maneuver for different road conditions. Studies have confirmed that the sensitivity of yaw moment with respect to AFS and DYC controls varies with vehicle stability regions [91, 106-108]. Eman et al. [91] investigated an integral and nonsingular fast terminal sliding mode control strategy to enhance the responses of vehicle side slip angle and yaw rate. To determine the respective working ranges of the AFS and the DYC, the sideslip angle and sideslip angular velocity phase plane was used. It is widely accepted that the mentioned phase plane boundaries are related to vehicle speed, road adhesion and front wheel steering angle; however, in order to reduce real-time computational burden, the variables used to describe the stability boundaries are often selected as constants [109]. For example, $\mathrm{Hu}$ et al. [92] proposed a two-layer hierarchical control structure based on SMC to improve vehicle handling stability, in which the operation region was dependent on tire slip angle.

Considering that the uncertainty of cornering stiffness often leads to the nonlinearity of tire forces, Yang et al. [98] proposed a new coordination scheme for AFS and DYC based on LQR, in which the cornering stiffness was treated as the function of road adhesion conditions. The phase plane of the side slip angle and side slip angular velocity decides the operating authority of the two systems and what kind of coordination control would be activated when the vehicle is considered as unstable. Generally, various operation region representing methods have been developed based on nonlinear tire forces, and recent studies have presented plenty of methods to fit the tire force in the nonlinear region 
[96, 110, 111]. Choi [96] developed an MPC-based coordinated control algorithm for AFS and ESC. The bicycle model was extended by linearizing the tire forces at operating points to capture the lagging characteristics. However, this showed poor fitting precision in the nonlinear region. Song et al. [112] developed a polygonal simplification method for tire forces allocation to deal with the constraints of tire adhesion limits while minimizing the working loads of four wheels. The proposed terminal sliding mode control architecture could effectively solve the understeer and oversteer problems.

A 4WS vehicle is capable of better handling performance and stability compared to a front-wheel-steered vehicle due to the availability of rear wheel steering [113, 114], especially in critical conditions [115]. Ono et al. [116] proposed an integrated control algorithm for 4WS and 4WD using an online nonlinear optimization approach. By minimizing the working loads of four wheels, the generalized forces are distributed according to the friction circle of each wheel. Ahmadi et al. [117] developed an integrated adaptive nonlinear control scheme to enhance the maneuverability and lateral stability. The control targets are the slip ratio and slip angle of each wheel. The rate of change of the tire force limited by the characteristics of actuators is also considered into the constraints. However, when referring to lateral dynamics, it is conventional to assume the longitudinal vehicle velocity as a constant, which is unreasonable in ever-varying driving scenarios [118, 119]. In Ref. [120], the vehicle longitudinal dynamics as well as the lateral and yaw motions is controlled simultaneously. A chattering-free variable structure controller utilizes the measured vehicle velocities in both longitudinal and lateral directions and yaw rate to calculate the steering angle and driving force of each wheel.

With the continuous focus on autonomous driving technology, full X-by-wire chassis platforms have raised more attention due to its strong ability of performing motion control during path tracking [33, 121, 122]. The motion control, as an essential part of path tracking, takes the waypoints information including both location information and speed profile as inputs and the generated control commands for the involved chassis subsystems as outputs. Yang et al. [99] proposed a hybrid fault-tolerant path tracking algorithm based on the linear-quadratic control method and the Lyapunov function. Through considering input constraints, actuator faults and external disturbances, the proposed scheme constructed a cost function to minimize the path tracking error. During motion control, path tracking accuracy relies heavily on the underlying vehicle model. A high-precision vehicle model with multiple degrees of freedom can comprehensively reflect vehicle dynamics response during path tracking; however, this leads to higher computational burden [90]. In Ref. [123], a full tenth-order vehicle model and a conventional simplified bicycle model were compared with respect to path tracking performance. It concluded that the bicycle model consumed less computational resources, but rendered poor tracking accuracy in high speed driving scenarios. Similarly, the work in Ref. [124] confirms the finding. Hence, a good tradeoff between model precision and computational burden always leads to the algorithm's robustness. Without the need of precise modeling, Zhu et al. [125] proposed a particle swarm optimization-based nonlinear predictive control scheme to execute coordinated control of steering and braking systems. This exhibited better robustness. Similarly, through introducing a parameters adjusting law based on the Lyapunov function, the technical research in Ref. [122] could better handle the unknown and nonuniform road conditions.

Accelerating/decelerating and steering are common maneuvers performed by drivers. Recent studies showed that their coordination control can give rise to substantial performance improvement under the scenarios such as emergency obstacle avoidance in autonomous driving [126-129], tire blowout control [130], anti-roll dynamics control [72, 77] and regenerative braking [124, 125]. An adaptive nonlinear coordinated control strategy based on SMC was developed to realize obstacle avoidance in Ref. [126]; however, it ignored the constraint of the maximum braking force determined by road adhesion condition and vehicle vertical motion. To solve this issue, Mehdi et al. [109] addressed a fuzzy-scheduled integrated control method for braking and steering systems to handle hard brakings during cornering, where the maximum braking force was determined by an additional wheel slip controller. However, the rollover risk in emergency scenarios was not considered [131-133]. When vehicle rollover occurs, it tends to be at high speed and under small road curvature, which would result in a large lateral acceleration. To be specific, as vehicle roll motion is directly related to the coupling relationship between tire and vehicle dynamics, multiple constraints in vehicle chassis control must be imposed to prevent vehicle rollover [134], such as the front wheel steering angle and rollover index limits. Hence, current literatures have been dedicated to preventing rollover through steering [135] and braking [136] control, or the combination of the two [137]. Imine et al. [135] proposed a SMC-based steering controller for rollover prevention by estimating vehicle CG height and suspension parameters. Similarly, Rajesh et al. [136] discussed the possibility of rollover avoidance via differential braking, which reduced 
vehicle speeds before sharp cornering. However, the above two ways for anti-rollover are faced with the problem of insufficient path tracking accuracy or conflicting with the desired vehicle motion. As supplementary, an integrated steering and braking coordinated control scheme for anti-roll over was developed in Ref. [137] to enhance vehicle stability and minimize path tracking error, where the rollover was prevented by limiting the steering angle and distributing the braking force.

In Ref. [72], the combined control of lateral and roll dynamics was achieved by active braking and steering. To this end, the roll state was measured by the Timeto-Stability (TTS) index. Regenerative braking can substantially reduce energy consumption and promote driving range per charge. By considering the responses of steering and braking subsystems, the work in Ref. [102] addressed the coordination of vehicle stability and regenerative braking.

\subsection{Suspension and Steering Coordinated Control}

In a critical lane change or sharp steering circumstance, the vehicle may roll over due to large lateral acceleration and suspension forces, which would lead to the reallocation of vertical loads on the tires. This will affect vehicle longitudinal and lateral dynamics. The vertical load transfer can be formulated as

$$
\begin{aligned}
& F_{z, f l}=\frac{m g b}{2 L_{w}}-\frac{m a_{x} h_{g}}{2 L_{w}}-\frac{m a_{y} h_{g}}{L_{w}} \frac{b}{L_{w}}, \\
& F_{z, f r}=\frac{m g b}{2 L_{w}}-\frac{m a_{x} h_{g}}{2 L_{w}}+\frac{m a_{y} h_{g}}{L_{w}} \frac{b}{L_{w}}, \\
& F_{z, r l}=\frac{m g a}{2 L_{w}}+\frac{m a_{x} h_{g}}{2 L_{w}}-\frac{m a_{y} h_{g}}{L_{w}} \frac{a}{L_{w}}, \\
& F_{z, r r}=\frac{m g a}{2 L_{w}}+\frac{m a_{x} h_{g}}{2 L_{w}}+\frac{m a_{y} h_{g}}{L_{w}} \frac{a}{L_{w}},
\end{aligned}
$$

where $a_{x}$ and $a_{y}$ are the longitudinal and lateral accelerations, respectively; $h_{g}$ is the vehicle CG height and $F_{z, i j}$ $(i j=f l, f r, r l, r r)$ refers to the vertical load of each wheel, which is highly related to the longitudinal and lateral tire forces. Take the Magic Formula for example, the nonlinear tire force can be given by

$$
Y(x)=D \sin \{C \arctan [B x-E(B x-\arctan B x)]\},
$$

where $Y(x)$ can represent the longitudinal or lateral tire force; $x$ is the tire slip angle or the vehicle longitudinal slip ratio; $B, C, D$, and $E$ are the shape parameters determined by vertical tire load and road adhesion coefficient.

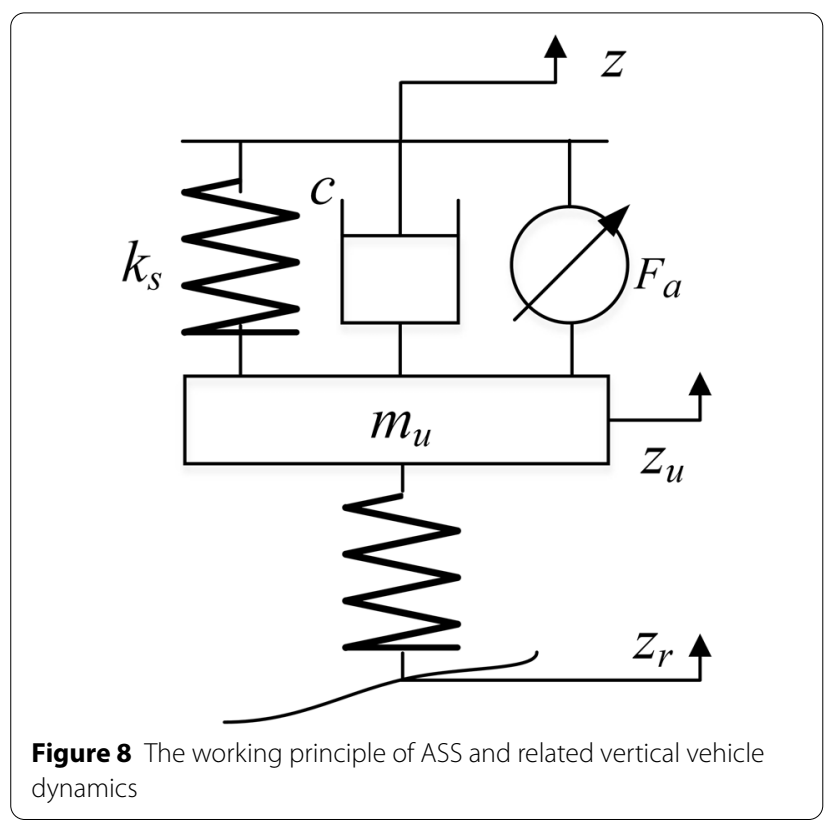

In normal driving scenarios, most studies ignore suspension vertical dynamics under the assumption that vehicle roll angle always remains within an acceptable range. Also, little attention is paid to the contribution of suspension to ride comfort and handling stability. In extreme driving scenarios such as high-speed cornering, the vertical load of tires changes sharply, which would result in high rollover risk. Recent work has developed various vehicle safety technologies to prevent rollover such as Active Roll Control System (ARS), Semiactive Suspension (SAS) and ASS. ARS can improve antirollover stability through actively controlling of front/ rear roll stiffness distribution ratio [138]. This is effective in reducing vehicle roll angle, especially in emergency cornering scenarios. An SAS system can make adjustments to the damping parameters corresponding to vehicle operating conditions. Devices that use an SAS system can provide energy and active control forces for being included in an ASS [139]. The spring stiffness and damping coefficients and the vehicle body height can be changed with respect to vehicle state and external disturbances. A simplified structure of ASS is shown in Figure 8.

Considering vehicle pitch and roll motions, the dynamics equations can be expressed as

$$
I_{y} \ddot{\theta}+\left(F_{v, f_{l}}+F_{v, f r}\right) \cdot a-\left(F_{v, r l}+F_{v, r r}\right) \cdot b=0,
$$




$$
\begin{gathered}
I_{x} \ddot{\phi}+\left(F_{v, f r}+F_{v, r r}\right) \cdot \frac{L_{w}}{2}-\left(F_{v, f l}+F_{v, r l}\right) \cdot \frac{L_{w}}{2}+ \\
m g h_{g} \sin \phi+m a_{y} h_{g} \cos \phi=0,
\end{gathered}
$$

where

$$
\begin{aligned}
& F_{v, f l}=k_{s, f l}\left(z_{f_{l}}-z_{u, f l}\right)+c_{f_{l}}\left(\dot{z}_{f l}-\dot{z}_{u, f l}\right)-F_{a, f l}-\frac{k_{f}}{L_{w}}\left(\phi-\frac{z_{u, f l}-z_{u, f r}}{L_{w}}\right), \\
& F_{v, r l}=k_{s, r l}\left(z_{r_{l}}-z_{u, r l}\right)+c_{r_{l}}\left(\dot{z}_{r l}-\dot{z}_{u, r l}\right) \quad-F_{a, r l}-\left\{\frac{k_{r}}{L_{w}}\left(\phi-\frac{z_{u, r l}-z_{u, r r}}{L_{w}}\right),\right. \\
& F_{v, r r}=k_{s, r r}\left(z_{r_{r}}-z_{u, r r}\right)+c_{r r}\left(\dot{z}_{r r}-\dot{z}_{u, r r}\right) \quad-F_{a, r r}-\frac{k_{r}}{L_{w}}\left(\phi-\frac{z_{u, r l}-z_{u, r r}}{L_{w}}\right), \\
& F_{v, f r}=k_{s, f r}\left(z_{f_{r}}-z_{u, f r}\right)+c_{f r}\left(\dot{z}_{f r}-\dot{z}_{u, f r}\right)-F_{a, f r}-\frac{k_{f}}{L_{w}}\left(\phi-\frac{z_{u, f l}-z_{u, f r}}{L_{w}}\right),
\end{aligned}
$$

where $F_{v, i j}$ and $F_{a, i j}(i j=f l, f r, r l, r r)$ are the vertical and the active suspension force, respectively; $k_{s, i j}$ and $c_{i j}$ are the spring stiffness and the damping coefficient of the ASS, respectively; $z_{i j}, \mathrm{z}_{u, i j}$ and $\mathrm{z}_{r, i j}$ refer to the sprung mass deflection, unsprung mass deflection and road excitation, respectively; $\theta, \phi$, and $I_{y}$ refer to the pitch angle, roll angle, and rotational inertial of the vehicle, respectively.

When a vehicle follows a curved trajectory, uncontrollable disturbances may affect steering angle due to vehicle roll motion, which is called roll steer. Basically, vehicle roll motion would impact steering kinematics parameters and then vehicle lateral stability and yaw dynamics due to the coupling mechanisms [140]. Moreover, due to the strong coupling relationship between the vertical and the lateral motion, when the vehicle turns, the vertical load transfer on the tires caused by lateral acceleration will directly affect the nonlinear tire force dynamics. This means the maximum lateral force and the yaw stability will be limited, possibly resulting in vehicle performance degradation. Further, in terms of the tireroad effect coupling, there are contradictions between the lateral and the roll stability [141]. High adhesion capacity tires usually contribute to better performance in lateral stability control but also have high rollover propensity. Additionally, the coupling effect between the vertical load transfer and the lateral and roll stability is a highly nonlinear process related to tire dynamics, chassis characteristics, and road excitations, etc. Therefore, the integrated control of steering and suspension systems has garnered extensive research interest. This seeks to enhance vehicle lateral and roll dynamics and coordinate different control objectives, especially in extreme circumstances. Figure 9 illustrates a widely accepted coordinated control architecture. This usually contains a reference model, a vehicle state estimator, a coordinated controller and several actuator models. enhance overall riding performance, a variable-geometry suspension system was developed in Ref. [142] considering independent steering.

In a steady-state operating condition, all the tires work in the linear region and the coupling relationship between vehicle vertical and lateral dynamics is weak. Hence, ASS mainly improves the steering status (understeer and oversteer), handling stability and ride comfort. Mehmet et al. [143] proposed a SMC-based integrated chassis controller for 4WS and active hydro-pneumatic suspension. The overall control scheme was decoupled, which is effective in linear operating conditions, i.e., for lateral accelerations below $4 \mathrm{~m} / \mathrm{s}^{2}$. To enhance yaw stability, March et al. [144] introduced an integrated control strategy for AFS and ASS to track the desired vehicle yaw rate. Similarly, Bei et al. [145] addressed an integrated control scheme using a fuzzy-Proportion-Integral-Derivative (PID) algorithm based on ESP and SAS. The auxiliary steering moment and the damping force are independently controlled to enhance overall vehicle dynamics response. However, the working regions of the EPS and SAS were not properly defined.

The tire slip angle tends to be small in steady-states, so many studies introduced a linear tire model to represent longitudinal and lateral tire forces. However, when the vehicle reaches its handling limits due to tire force saturation, describing the complex tire forces becomes difficult. Compromise between model precision and computational burden is usually necessary. Moreover, rollover becomes a noticeable factor that impacts vehicle active safety in some emergency driving scenarios. Hence, active suspension control systems prioritize anti-roll and enhance vehicle safety primarily in potential rollover conditions [146-148]. Imine et al. presented a sliding mode-based steering controller to reduce load transfer and therefore to stabilize a heavy-duty vehicle 


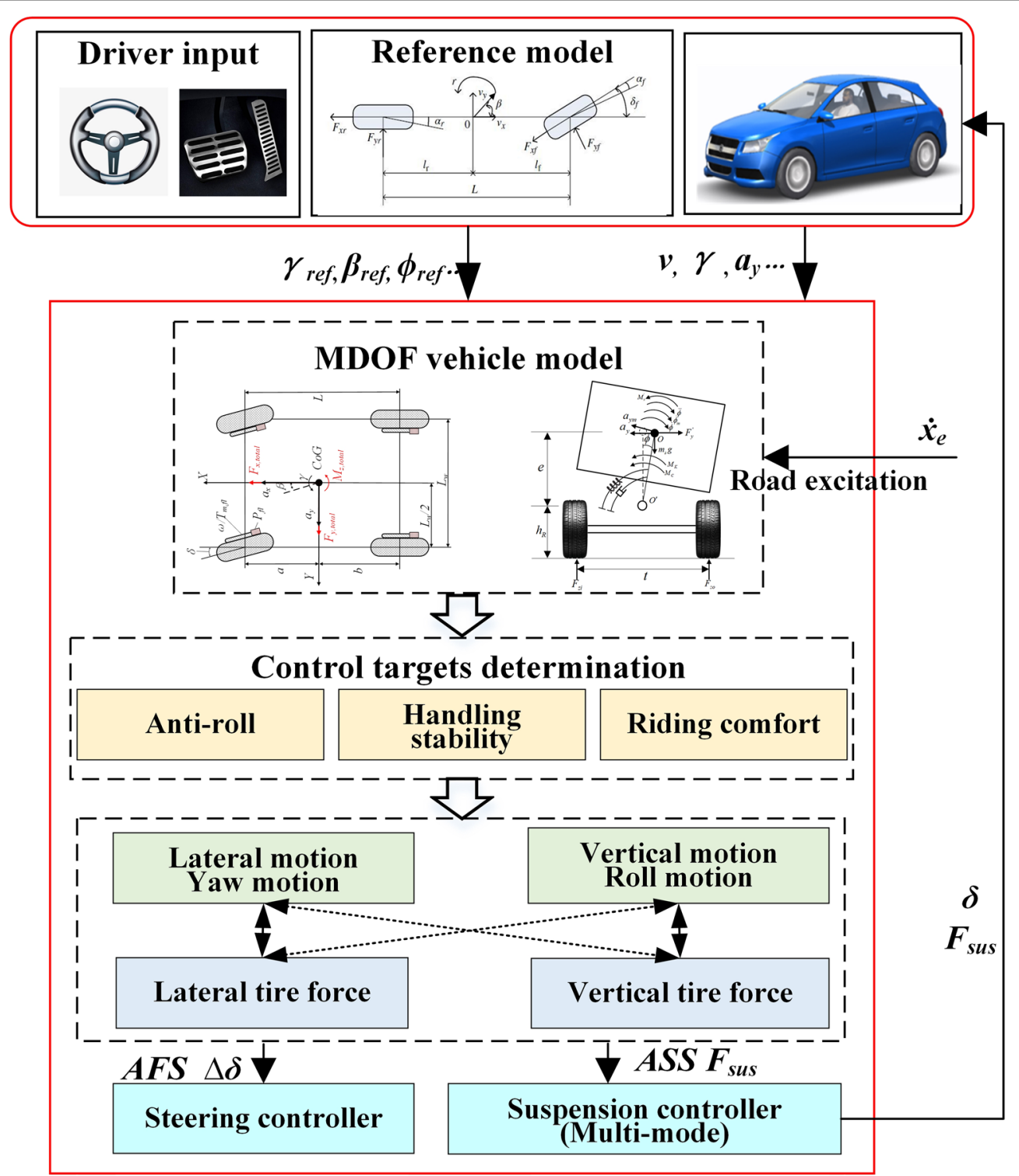

Figure 9 Coordinated control of steering and suspension systems

[146]. The LTR was utilized to represent the roll state and the lateral acceleration was limited by the steering controller based on the LTR metering. Dahmani et al. [149] developed an observer-based state feedback tracking controller using 4WS and ASS to regulate vehicle lateral motion and load transfer during extreme conditions. To accurately capture vehicle lateral and roll dynamics, a model-based observer was introduced to estimate the vehicle roll angle and the tire slip angle. Furthermore, a Takagi-Sugeno (TS) representation of lateral tire force was utilized to describe the nonlinear characteristics of tires in emergency conditions. The steering angles at both axles and the active roll moment produced by the ASS guarantee a good tracking of the desired side slip angle, yaw rate and load transfer of the vehicle. However, the trigger rule was not explained clearly. In Ref. [150], the development of a conventional yaw stability judgement scheme led to the introduction of a hierarchical control scheme for AFS and ASS. These used the stability region boundaries which were determined from a phase plane of vehicle side slip angle and its rate of change. The coordinated control of AFS and ASS is activated only when the vehicle reached the unstable region. Moreover, switching rollover controller designs for multi-mode suspension control, the performance index, and multi-objective cost functions were comprehensively discussed in Refs. [147, 


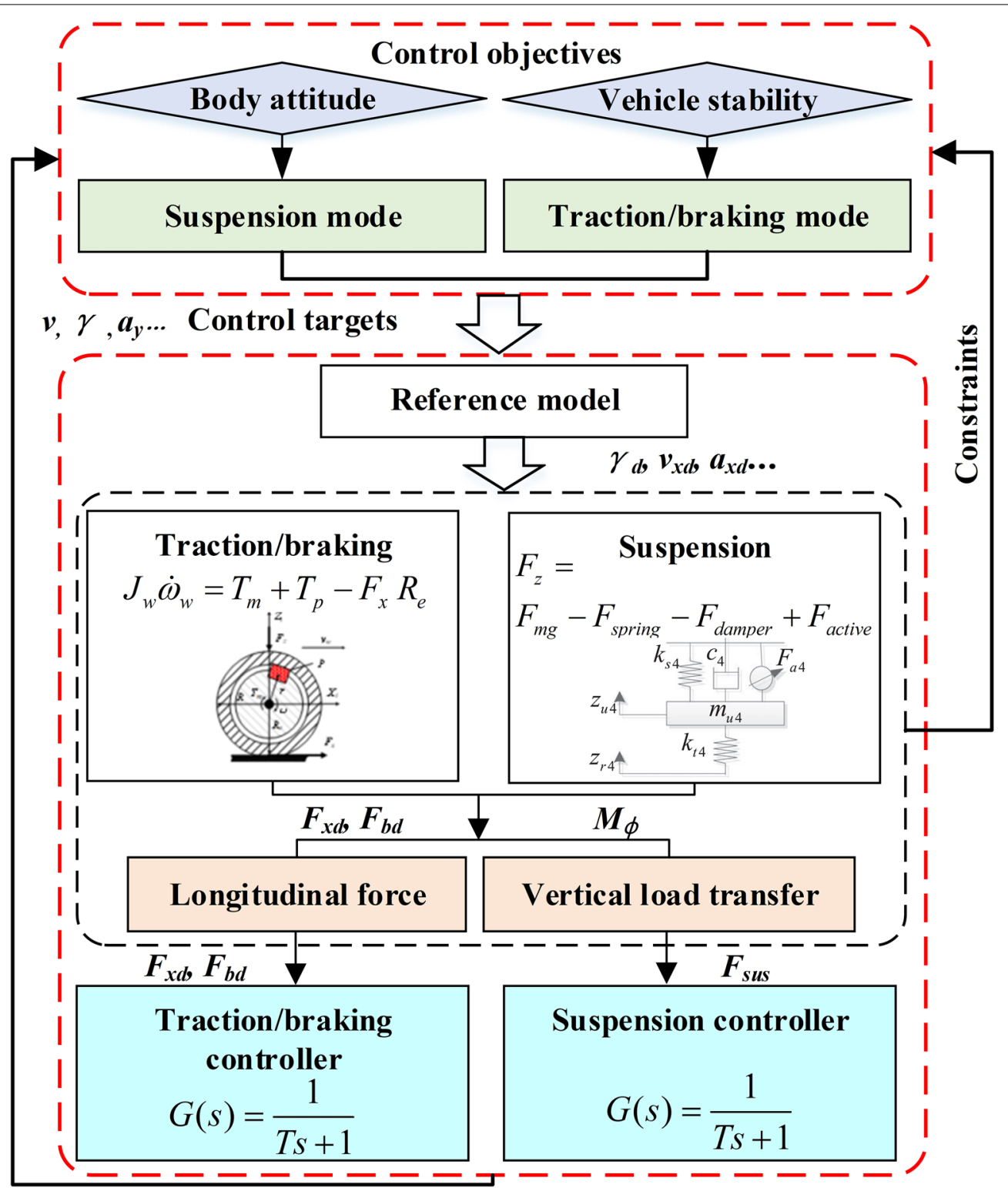

Figure 10 Coordinated control of driving/braking and suspension systems

148] to fully consider the ride comfort, handling stability and anti-rollover performance of a vehicle equipped with steering-by-wire and suspension-by-wire systems.

\subsection{Suspension and Driving/Braking Coordinated Control} It is widely accepted that when an emergency braking or accelerating maneuver is performed in a vehicle, the maximum ground driving force, which is closely related to road adhesion coefficient and tire vertical load, must be guaranteed. The vertical load of tires can be adjusted according to driving conditions and vehicle states if the vehicle is equipped with a wire-controlled suspension system. Hence, as shown in Figure 10, the integrated control of the active suspension and the driving/braking system has the potential for active safety control of vehicle, especially in emergency scenarios. The advantages of body altitude control, vibration attenuation and ride comfort optimization should not be ignored. Much research has focused on the evaluation of onboard vibration and ride comfort for passengers. Research has shown that the accelerations caused by vertical vibration contribute to an essential part of uncomfortableness of passengers. Therefore, under the premise of ensuring safety, how to optimize ride comfort via coordinated control of 
driving/braking and suspension systems has attracted much interest.

The most mature active safety systems in braking systems are ABS and ESC. ABS prevents the rotating wheels from locking by applying braking forces to each individual wheel to obtain their respective optimal slip ratio. This means that acquiring real-time slip ratio and road adhesion coefficient is vital in developing an enabling ABS control strategy. ESC is an combination of ABS and TCS. This enhances vehicle lateral stability and prevents the vehicle from severe understeer and drifting. This is done using active driving or braking forces for different wheels. It is worth mentioning that the combination of ESP and ASS performs well in preventing rollover while ensuring vehicle yaw stability. This is especially true for Sport Utility Vehicles which have higher CGs.

Many studies have demonstrated that the combination of ABS and ASS can effectively shorten braking distance and enhance ride comfort during braking events [151153]. Wang et al. [152] proposed an integrated algorithm combinatively using suspension and braking forces to reduce the braking distance. The ASS was utilized to obtain the desired vertical force profile during a braking maneuver. When hard braking operations are detected, the increased tire vertical force generated by the ASS can provide larger braking force to reduce the braking distance. However, the simplified vehicle model only focuses on the vehicle longitudinal and the suspension vertical dynamics [154]. This means that lateral dynamics is neglected in the algorithm, which leads to limited performance during steering maneuvers.

A new way of controlling the peak tire-road adhesion coefficient within a stable area based on the barrier Lyapunov function to reduce braking distance was put forward in Ref. [155], which is different from conventional methods that treat the reference slip ratio as a control objective. However, road adhesion condition is one of the key factors that determine the optimal slip ratio, which indicates that accurate road adhesion coefficient estimation is necessary for an enabling ABS system. Wang et al. [156] designed an observer-based direct adaptive fuzzy neural controller for the coordination of ABS and ASS, in which the LuGre friction model was utilized in the road adhesion estimator. This can transmit the reference slip ratio to the ABS controller through a mapping function of road surface characteristics, and also considers fault tolerant control against uncertainty and failure for the ASS. However, if the system dynamics is unavailable, the efficiency of the mentioned DFAC algorithm would be low. To solve the uncertainties with braking and suspension systems, Wang et al. [152] addressed a hierarchical TS fuzzy neural model to identify unknown system dynamics and reduce the fuzzy rules and computational time.

In addition to transmitting the forces and moment between the wheels and the vehicle frame, an essential function of suspension system is to cushion the impact force caused by road excitations as well as to attenuate the vibration to ensure riding comfort of passengers. But riding discomfort brought by emergency braking is usually ignored. To tackle this drawback, Wu et al. [157] developed an intelligent car following model for ride comfort improvement. A phase plane that considered the speed and distance between the host and the preceding vehicle was used to assess ride comfort. A reference deceleration profile was applied to the host vehicle using the relationship between braking safety and ride comfort. However, the proposed car following model only demonstrated its effectiveness in straight running. Recently, integrated control of ESP and ASS has been shown to be advantageous in ensuring ride comfort and driving stability $[158,159]$. This includes steering scenarios where the vehicle is not simply moving in a straight line. Xiao et al. [158] developed a hierarchical control method for ESP and ASS, which simultaneously enhanced vehicle handling stability and ride comfort. In the scheme, the upper layer controller was responsible for coordinating the EPS and the ASS. This achieves an ideal distribution of the total yaw moment derived from a reference bicycle model. The Linear-Quadratic-Gaussian control and the adaptive fuzzy logic were applied to the individual controllers of the ASS and ESP, respectively. This was to track the desired yaw rate and side slip angle of the vehicle. However, the weighting coefficients for yaw moment distribution were set and adjusted by experience, resulting in poor robustness and applicability when facing diverse driving conditions. The trigger logic and working regions of the subsystems were not defined comprehensively so that all the chassis subsystems must work in all conditions. This is challenging for the design of the MultiInput-Multi-Output (MIMO) controller and the overall performance of the integrated algorithm. Chu et al. [160] proposed a rule-based coordinated control strategy for ABS and ASS. The longitudinal acceleration and pitch angle of vehicle were used to determine the intervention conditions of the proposed algorithm. However, actuator constraints and possible time relays were not fully considered. Zhang et al. [161] developed a switching strategy for ASS control to assist an ABS. This enhanced the braking performance while improving ride comfort. The suspension displacement is used to determine the working mode of the ASS while the actuator constraints were used to limit the control outputs. However, as the actual braking situation is ignored, its effectiveness needs 
verifications on the roads with time-varying roughness. Following on from Ref. [161], Vassal et al. [162] used the real-time slip ratio of tires and the braking force of vehicle as the criteria to determine whether the overall control target is active safety or ride comfort.

Both differential braking and ASS can prevent vehicle rollover. The former focuses on reducing vehicle yaw rate and lateral acceleration while the latter prioritizes limiting the LTR, both through adjusting spring and damper parameters. However, if only differential braking is used, this will lead to poor maneuverability and path tracking performance. ASS is limited in attenuating the impact brought by large lateral accelerations. Therefore, the anti-roll potential of integrated control of the driving/ braking and suspension systems has attracted interest. Chou et al. [163] designed a wholistic vehicle control strategy using differential braking and active suspension based on the nonlinear constrained optimization and the singular perturbation theory. The overall target was to track the generated path using longitudinal vehicle speed and yaw rate. Meanwhile, the differential braking and the active suspension were utilized to restrict the roll and vertical accelerations of the vehicle body. As previously discussed, the involvement of the ASS would obviously impact vehicle steering characteristics. To deal with it, Yoon et al. [164] developed an unified chassis control strategy using ESP and the Continuous Damping Control (CDC) to prevent vehicle rollover and enhance lateral stability. A model-based estimator was used to obtain the real-time roll angle and the Rollover Index (RI) was utilized to quantitatively gauge the risk of vehicle rollover.

\subsection{Coordination of Three Subsystems}

As previously discussed, most of chassis subsystem controllers are designed for independent control functions and optimized for specific operating regions, so they can only influence vehicle dynamics to quite a limited extent. However, there are high coupling relationships between longitudinal, lateral, and vertical tire forces and then individual chassis subsystems. The participation of any chassis subsystem would affect the overall chassis dynamics. Independent controllers for chassis subsystems have been well researched [165]; but they are always faced with time-varying driving conditions and conflicting control objectives. Thus, it is important to develop a hierarchical control strategy that contains all the chassis subsystems to obtain globally optimal vehicle dynamcis response in diverse driving conditions.

Vehicle chassis coordinated control has been an important area of research over the last few decades. For instance, Bosch developed the Vehicle Dynamic Management (VDM) using active braking, steering and suspension systems. Similarly, Delphi designed the
Unified Chassis Control (UCC). The strong coupling relationship and nonlinearity of tire forces were used to develop efficient control schemes. This can unify the control targets for different driving scenarios and eliminate potential conflicts for different actuators. For lateral stability, both steering and driving/braking systems can generate a corrective yaw moment directly or indirectly via changing the front wheel steering angle or the longitudinal traction/braking forces on individual wheels of IWMD EVs $[166,167]$. ASS has demonstrated its effectiveness in adjusting vertical dynamics as well as reducing braking distance and understeer/oversteer tendency. Under critical driving circumstances, it can prevent vehicle rollover through adjusting roll stiffness or applying active suspension forces. Path tracking performance and yaw dynamics stability can be enhanced by steering and traction/braking systems. Moreover, through adjusting longitudinal, lateral, and vertical forces, the desired vehicle body motion including pitch, roll, and yaw could be achieved [168].

Recently, hierarchical control architecture has been widely adopted because of its modular structure, robustness, and optimality for the control allocation paradigm. This can be clearly seen in Figure 11 [112, 138, 169-173]. Using the interface correspondence between different controller levels, the hierarchical scheme can reduce the complexity of controller design and the coupling relationships of various chassis subsystems [174]. In this sense, Li et al. [169] proposed a main/servo-loop structure consisting of 4WS, DYC and an active stabilizer to enhance vehicle lateral and roll stability. A SMC-based main-loop controller was utilized to obtain the generalized forces and moment using a reference vehicle model. The servo-loop controller was responsible for control allocation based on the Sequential Quadratic Programming approach. However, the obtained active anti-roll moment was simply based on a proportion function of lateral acceleration. Unfortunately, this gave a lagging control response and poor robustness as the suspension dynamics was neglected.

Cho et al. [170] developed an integrated control strategy for ESP, AFS and CDC to achieve target vehicle lateral and roll response. A coordinated control of AFS and ESP was used to obtain the optimal distribution of the generalized yaw moment. This reduced the longitudinal vehicle velocity loss. An SMC-based CDC controller was proposed to minimize the roll angle using lateral acceleration and sprung mass velocity. Noticeably, this work estimated the key parameters that heavily influence the overall control performance, i.e., the cornering stiffness and vertical load of tires.

Zhao et al. [175] addressed a three-layer hierarchical control strategy to coordinate the interactions of AFS, 


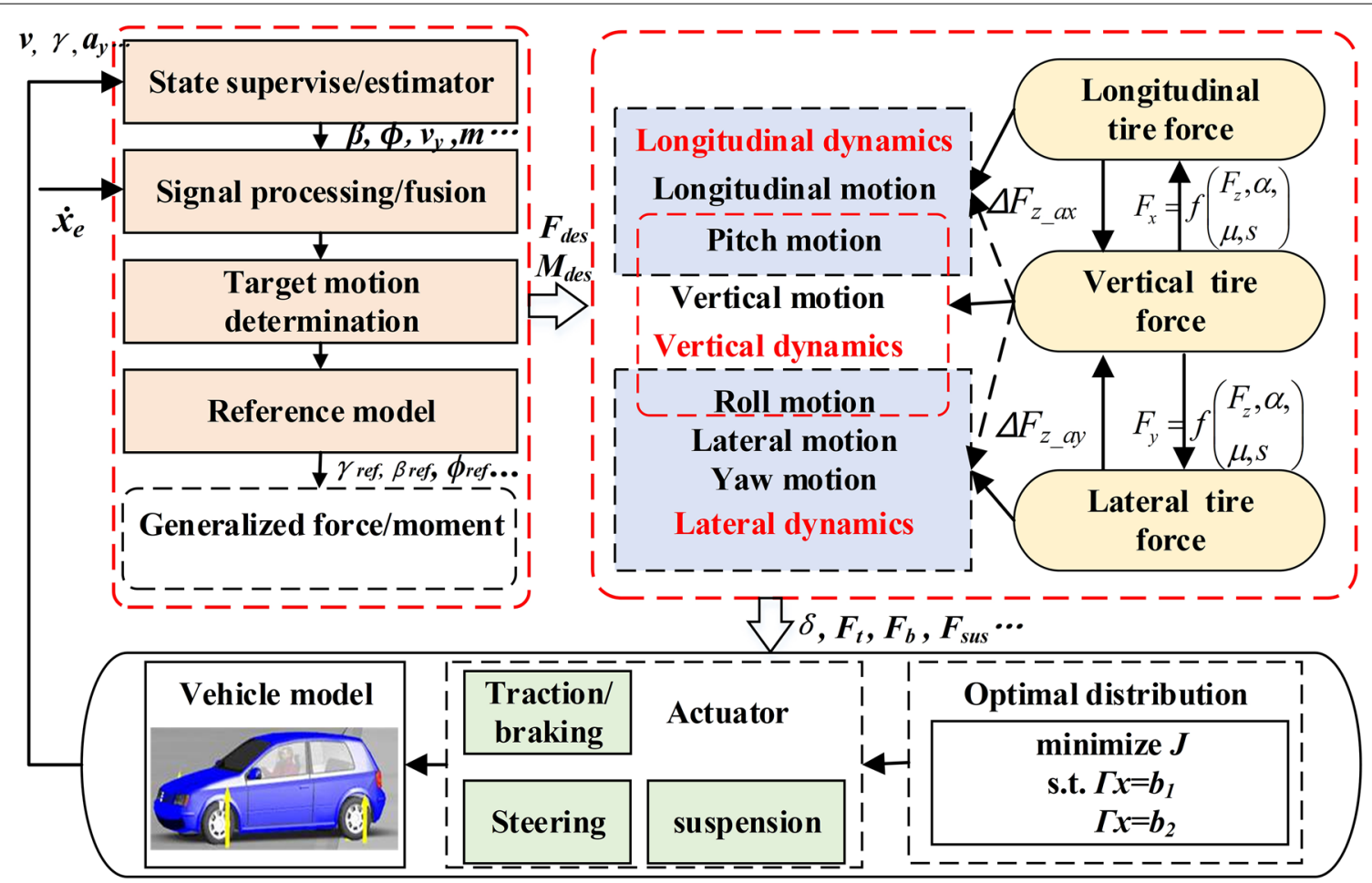

Figure 11 Coordinated control of three chassis subsystems

DYC and ASS. A Particle Swarm Optimization-based Fuzzy Logic Control algorithm was designed to distribute the yaw moment using operating regions and trigger conditions. The corrective moment generated by the ASS was determined by the weighting coefficient from the total corrective moment based on the trial and error method. However, it only focused on the lateral dynamics of the vehicle and overlooked the roll motion. As a development of Ref. [175], Ref. [138] acquired the desired roll moment using the target vehicle motion. A parameterdependent Linear Parameter Varying-based control allocation algorithm was adopted to achieve optimal lateral and roll stability. The continuous development of IWMD EVs has provided increased potential for chassis coordinated control by applying precisely controllable driving/ brake torques to individual wheels.

Song et al. [112] presented a novel integrated chassis controller with multiple hierarchical layers for a full $\mathrm{X}$-by-wire vehicle. This improved vehicle stability and handling performance. The attained generalized forces and moment were obtained using a terminal sliding mode controller in the motion control layer. They were distributed to individual wheels by the constrained tire loads allocator.

Vehicle driving conditions and control objectives vary frequently. Due to the multi-functional requirements of active safety, handling stability, maneuverability, and ride comfort, it is evident that an invariable control architecture can barely meet the requirements in all driving conditions [176]. Recently, researchers have validated that a multi-mode switching control strategy can improve the overall performance of a vehicle in various driving circumstances using driving condition identification as discussed in Section 2 [42, 171, 177, 178]. Lu et al. [42] proposed an SMC-based main-objectiveoriented two-layer hierarchy control method, in which the upper monitor layer was charged with identifying current driving condition through a set of state variables such as characteristic vehicle speed, steering wheel angle, vehicle yaw rate, as well as others. A rule-based classifier divided the driving conditions into seven state modes and accordingly different control patterns. For instance, the control priority under emergency braking scenarios would switch to safety rather than ride comfort.

Her et al. [177] developed an integrated optimal control scheme based on ESP, ARS and electronic control suspensions to improve vehicle agility and stability. Using the lateral acceleration and steering status of the vehicle, the vehicle operating region was divided into three modes: the agility mode, the maneuverability mode and the stability mode. Similarly, in Ref. [178], the driving 
conditions were classified into three categories by using steering status and braking intensity to evaluate the emergency level of current driving condition.

Much work has been devoted to improving vehicle roll stability using chassis coordinated control [172, 179]. Lu et al. [179] discussed the authority and effective working regions of the steer, brake and suspension systems for rollover prevention. This work used the fuzzy logic and shows that the AFS system possesses the largest control authority and the active braking system has the largest effective region. The study in Ref. [172] provided a unique method for rollover control via ASS. This has two sets of control parameters that are appropriate for different working regions for the vehicle.

\section{Conclusions}

This paper has presented a comprehensive and systematic review on chassis coordinated control for full X-by-wire vehicles. Much work has been carried on this regard. The following points can be made.

(1) For driving condition identification, the simple rule-based method shows the advantage of low computational burden while model- and neural network-based methods possess high precision and robustness. The combination of different kinds of methods is expected to satisfy the requirements of real-time implementation and high robustness in varied driving conditions.

(2) For chassis coordinated control, Active Front Steering (AFS) can effectively generate the desired yaw moment through steering angle compensation when the side slip angle is small. In contrast, Direct Yaw-Moment control (DYC) is capable of directly interfering in vehicle yaw motion by applying driving/braking forces to individual wheels even when the vehicle operates in the nonlinear region. The real-time and optimal allocation of the desired yaw moment between AFS and DYC has been the subject of many studies.

(3) Vehicle roll motion is often ignored when lateral acceleration is small; however, the load transfer caused by vehicle roll motion would heavily influence the overall longitudinal and lateral forces acting on the vehicle and the cornering stiffness of tires. Therefore, vehicle roll and vertical dynamics are noticeable in critical circumstances. Using vehicle roll status identification, the control authority between Active Suspension System and differential braking should be further investigated.

(4) The effectiveness of a hierarchical control strategy with multi-functional control modes has been demonstrated. The monitor layer usually uses the rules and the thresholds of critical vehicle states for identifying current driving condition. This should be further discussed. Moreover, for real-time applicability, the event trigger theory may be a promising solution [180].

(5) The actuators characteristics of the chassis require full consideration. The dynamic response and time relays are rarely considered in chassis coordinated control synthesis. For a control strategy with multimodes, frequent switchings between different control modes is still challenging in terms of robust design.

(6) For autonomous vehicles, the ideal speed, acceleration and yaw rate of vehicle and the road curvature can be generated in the trajectory planning module. This is heavily related to driving condition identification. Hence, incorporating trajectory planning may considerably contribute to driving condition identification and chassis coordinated control for full X-by-wire vehicles. Besides, implementing chassis coordinated control in the path tracking needs to be further investigated.

(7) Future research directions include coordinated control under critical driving conditions, fault-tolerant control against sensor and actuator failures and combinative synthesis with motion planning.

\section{Authors' contributions}

LZ was in charge of conceptualization, data analysis and manuscript writing and revisions; ZZ collected and analyzed the literature and prepared the draft; ZW and JD assisted with the manuscript revising and provided consultations; DGD revised the manuscript and gave suggestions. All authors read and approved the final manuscript.

\section{Authors' Information}

Lei Zhang, born in 1987, received the Ph.D. degree in Mechanical Engineering from the Beijing Institute of Technology, China, and the Ph.D. degree in Electrical Engineering from the University of Technology, Sydney, Australia, in 2016. He is now an Associate Professor with School of Mechanical Engineering, Beijing Institute of Technology, China. His research interests include management techniques for energy storage systems and vehicle dynamics and advanced control for intelligent electrified vehicles.

Zhiqiang Zhang, born in 1996, is currently pursuing the Ph.D. degree in Mechanical Engineering with National Engineering Laboratory for Electric Vehicles, Beijing Institute of Technology, China. His research interests include vehicle dynamics and active safety control for intelligent electrified vehicles.

Zhenpo Wang, born in 1976, received the Ph.D. degree in Automotive Engineering from Beijing Institute of Technology, China, in 2005. He is currently a Professor with Beijing Institute of Technology, China, and the Director of National Engineering Laboratory for Electric Vehicles.

Junjun Deng, born in 1985, received the B.S., M.S., and Ph.D. degrees in electrical engineering from Northwestern Polytechnical University, China, in 2008,

2011, and 2015, respectively. He is currently an Associate Professor with School of Mechanical Engineering, Beijing Institute of Technology, China. 
David G. Dorrell, has a BEng (Hons) from The University of Leeds (1988), MSC from The University of Bradford (1989) and PhD from The University of Cambridge (1993). He is currently a Distinguished Professor with The University of the Witwatersrand. He was Professor of Electrical Machines with The University of KwaZulu-Natal in Durban, South Africa (2015-2020) and Director of the EPPEI Specialization Centre in HVDC and FACTS at UKZN (2016-2020). He has held positions with The Robert Gordon University, UK, The University of Reading, UK, The University of Glasgow, UK, and the University of Technology Sydney, Australia. His research interests cover electrical machines, renewable energy and power systems. He has worked in industry and carried out several industrial consultancies. He is a Chartered Engineer in the UK and a Fellow of the IET.

\section{Funding}

Supported in part by Ministry of Science and Technology of the People's Republic of China (Grant No. 2017YFB0103600) and Beijing Municipal Science and Technology Commission via the Beijing Nova Program (Grant No. Z201100006820007).

\section{Competing interests}

The authors declare no competing financial interests.

\section{Author Details}

${ }^{1}$ National Engineering Laboratory for Electric Vehicles, Beijing Institute of Technology, Beijing 100081, China. ${ }^{2}$ Collaborative Innovation Center for Electric Vehicles in Beijing, Beijing Institute of Technology, Beijing 100081, China. ${ }^{3}$ School of Electrical and Information Engineering, University of the Witwatersrand, Johannesburg, South Africa.

Received: 18 December 2020 Revised: 9 March 2021 Accepted: 13 March 2021

Published online: 11 May 2021

\section{References}

[1] M Wang, Z Wang, L Zhang, et al. Speed planning for autonomous driving in dynamic urban driving scenarios. 2020 IEEE Energy Conversion Congress and Exposition (ECCE), Oct 11-15, 2020: 1462-1468.

[2] D Yang, S Zheng, C Wen, et al. A dynamic lane-changing trajectory planning model for automated vehicles. Transportation Research Part C: Emerging Technologies, 2018, 95: 228-247.

[3] L Thibault, G D Nunzio, A Sciarretta. A unified approach for electric vehicles range maximization via eco-routing, eco-driving, and energy consumption prediction. IEEE Transactions on Intelligent Vehicles, 2018, 3(4): 463-475.

[4] J E Siegel, D C Erb, S E Sarma. A survey of the connected vehicle landscape-Architectures, enabling technologies, applications, and development areas. IEEE Transactions on Intelligent Transportation Systems, 2018, 19(8): 2391-2406.

[5] A Sarker, H Shen, M Chowdhury, et al. A review of sensing and communication, human factors, and controller aspects for information-aware connected and automated vehicles. IEEE Transactions on Intelligent Transportation Systems, 2020, 21(1): 7-29.

[6] P Falcone, F Borrelli, J Asgari, et al. Predictive active steering control for autonomous vehicle systems. IEEE Transactions on Control Systems Technology, 2007, 15(3): 566-580.

[7] E Esmailzadeh, A Goodarzi, G R Vossoughi. Optimal yaw moment control law for improved vehicle handling. Mechatronics, 2003, 13(7): 659-675.

[8] L Zhai, T Sun, J Wang. Electronic stability control based on motor driving and braking torque distribution for a four in-wheel motor drive electric vehicle. IEEE Transactions on Vehicular Technology, 2016, 65(6): 4726-4739.

[9] J Zhang, Y Li, L Chen, et al. New regenerative braking control strategy for rear-driven electrified minivans. Energy Conversion and Management, 2014, 82: 135-145.

[10] J Cao, H Liu, P Li, et al. State of the art in vehicle active suspension adaptive control systems based on intelligent methodologies. IEEE Transactions on Intelligent Transportation Systems, 2008, 9(3): 392-405.
[11] Y Wang, Y Li, L Jiang, et al. PSO-based optimization for constantcurrent charging pattern for li-ion battery. Chinese Journal of Electrical Engineering, 2019, 5(2): 72-78.

[12] Y R Kafle, S U Hasan, G E Town. Quasi-Z-source based bidirectional DC-DC converter and its control strategy. Chinese Journal of Electrical Engineering, 2019, 5(1): 1-9.

[13] P Ning, $\mathrm{H} \mathrm{Li}$, Y Huang, et al. Review of power module automatic layout optimization methods in electric vehicle applications. Chinese Journal of Electrical Engineering, 2020, 6(3): 8-24.

[14] W Zhang, Y Xu, Y Huang, et al. Reduction of high-frequency vibration noise for dual-branch three-phase permanent magnet synchronous motors. Chinese Journal of Electrical Engineering, 2020, 6(2): 42-51.

[15] G K Singh, A lqbal. Small signal stability of three-phase and six-phase synchronous motors: A comparative analysis. Chinese Journal of Electrical Engineering, 2020, 6(1): 22-40.

[16] TKobayashi, E Katsuyama, H Sugiura, et al. Efficient direct yaw moment control: tyre slip power loss minimisation for four-independent wheel drive vehicle. Vehicle System Dynamics, 2017, 56(5): 719-733.

[17] E Joa, K Park, Y Koh, et al. A tyre slip-based integrated chassis control of front/rear traction distribution and four-wheel independent brake from moderate driving to limit handling. Vehicle System Dynamics, 2017, 56(4): 579-603.

[18] X Ji, X He, C Lv, et al. A vehicle stability control strategy with adaptive neural network sliding mode theory based on system uncertainty approximation. Vehicle System Dynamics, 2017, 56(6): 923-946.

[19] B Paden, M Cap, S Z Yong, et al. A survey of motion planning and control techniques for self-driving urban vehicles. IEEE Transactions on Intelligent Vehicles, 2016, 1(1): 33-55.

[20] B Okumura, M James, Y Kanzawa, et al. Challenges in perception and decision making for intelligent automotive vehicles: A case study. IEEE Transactions on Intelligent Vehicles, 2016, 1(1): 20-32.

[21] Y Luo, Y Xiang, K Cao, et al. A dynamic automated lane change maneuver based on vehicle-to-vehicle communication. Transportation Research Part C: Emerging Technologies, 2016, 62: 87-102.

[22] B Li, Y Zhang, Y Feng, et al. Balancing computation speed and quality: A decentralized motion planning method for cooperative lane changes of connected and automated vehicles. IEEE Transactions on Intelligent Vehicles, 2018, 3(3): 340-350.

[23] W Zhang, Z Wang, C Zou, et al. Advanced vehicle state monitoring: Evaluating moving horizon estimators and unscented Kalman filter. IEEE Transactions on Vehicular Technology, 2019, 68(6): 5430-5442.

[24] B L Pence, H K Fathy, J L Stein. Recursive estimation for reduced-order state-space models using polynomial chaos theory applied to vehicle mass estimation. IEEE Transactions on Control Systems Technology, 2014, 22(1): 224-229.

[25] L Y Hsu, T L Chen. Vehicle full-state estimation and prediction system using state observers. IEEE Transactions on Vehicular Technology, 2009, 58(6): 2651-2662.

[26] A A Brown, S N Brennan. Lateral vehicle state and environment estimation using temporally previewed mapped lane features. IEEE Transactions on Intelligent Transportation Systems, 2015, 16(3): 1601-1608.

[27] B L Boada, D G Pozuelo, M J L Boada, et al. A constrained dual Kalman filter based on pdf truncation for estimation of vehicle parameters and road bank angle: Analysis and experimental validation. IEEE Transactions on Intelligent Transportation Systems, 2017, 18(4): 1006-1016.

[28] KT Leung, J F Whidborne, D Purdy, et al. A review of ground vehicle dynamic state estimations utilising GPS/INS. Vehicle System Dynamics, 2011, 49(1-2): 29-58.

[29] K B Singh, M A Arat, STaheri. Literature review and fundamental approaches for vehicle and tire state estimation. Vehicle System Dynamics, 2018, 57(11): 1643-1665.

[30] X Jin, G Yin, N Chen. Advanced estimation techniques for vehicle system dynamic state: A survey. Sensors (Basel), 2019, 19(19).

[31] H Guo, Z Yin, D Cao, et al. A review of estimation for vehicle tire-road interactions toward automated driving. IEEE Transactions on Systems, Man, and Cybernetics Systems, 2019, 49(1): 14-30.

[32] T Nguyen, Y Wong, B Lechner. Response-based methods to measure road surface irregularity: A state-of-the-art review. European Transport Research Review, 2019, 11(1). 
[33] J Choi, KYi, J Suh, et al. Coordinated control of motor-driven power steering torque overlay and differential braking for emergency driving support. IEEE Transactions on Vehicular Technology, 2014, 63(2): 566-579.

[34] W Hsiao, M Horng, Y Tsai, et al. A driving behavior detection based on a zigbee network for moving vehicles. 2012 Conference on Technologies and Applications of Artificial Intelligence, Kaohsiung, Taiwan, 2012.

[35] D Mitrovic. Reliable method for driving events recognition. IEEE Transactions on Intelligent Transportation Systems, 2005, 6(2): 198-205.

[36] P Raksincharoensak, T Mizushima, M Nagai. Direct yaw moment control system based on driver behaviour recognition. Vehicle System Dynamics, 2008, 46(1): 911-921.

[37] D Tran, J Du, W Sheng, et al. A human-vehicle collaborative driving framework for driver assistance. IEEE Transactions on Intelligent Transportation Systems, 2019, 20(9): 3470-3485.

[38] B Wu, Y Chen, C Yeh, et al. Reasoning-based framework for driving safety monitoring using driving event recognition. IEEE Transactions on Intelligent Transportation Systems, 2013, 14(3): 1231-1241.

[39] V Leonhardt, T Pech, G Wanielik. Data fusion and assessment for maneuver prediction including driving situation and driver behavior. International Conference on Information Fusion, Heidelberg, Germerny, July 5-8, 2016 .

[40] M Bahram, C Hubmann, A Lawitzky, et al. A combined model- and learning-based framework for interaction-aware maneuver prediction. IEEE Transactions on Intelligent Transportation Systems, 2016, 17(6): 1538-1550.

[41] X Peng, Y L Murphey, R Liu, et al. Driving maneuver early detection via sequence learning from vehicle signals and video images. Pattern Recognition, 2020, 103.

[42] S B Lu, Y N Li, S B Choi, et al. Integrated control on MR vehicle suspension system associated with braking and steering control. Vehicle System Dynamics, 2011, 49(1-2): 361-380.

[43] B Higgs, M Abbas. Segmentation and clustering of car-following behavior: recognition of driving patterns. IEEE Transactions on Intelligent Transportation Systems, 2015, 16(1): 81-90.

[44] L He, H O Xiang, D X Chen, et al. Emergency obstacle avoidance control method based on driver steering intention recognition for steer-bywire vehicle. Advanced Materials Research, 2013, 694-697: 2738-2741

[45] S Sekizawa, S Inagaki, T Suzuki, et al. Modeling and recognition of driving behavior based on stochastic switched ARX model. IEEE Transactions on Intelligent Transportation Systems, 2007, 8(4): 593-606.

[46] Q Liu, S Xu, C Lu, et al. Early recognition of driving intention for lane change based on recurrent hidden semi-Markov model. IEEE Transactions on Vehicular Technology, 2020, 69(10): 10545-10557.

[47] C D'Agostino, A Saidi, G Scouarnec, et al. Learning-based driving events recognition and its application to digital roads. IEEE Transactions on Intelligent Transportation Systems, 2015, 16(4): 2155-2166.

[48] L Liu, Z Wang, S Qiu. Driving behavior tracking and recognition based on multisensors data fusion. IEEE Sensors Journal, 2020, 20(18): 10811-10823.

[49] Y Zheng, J H L Hansen. Lane-change detection from steering signal using spectral segmentation and learning-based classification. IEEE Transactions on Intelligent Vehicles, 2017, 2(1): 14-24.

[50] T Han, J Jing, U Ozguner. Driving intention recognition and lane change prediction on the highway. IEEE Intelligent Vehicles Symposium, Paris, France, June 9-12, 2019.

[51] Z Ouyang, J Niu, Y Liu, et al. An ensemble learning-based vehicle steering detector using smartphones. IEEE Transactions on Intelligent Transportation Systems, 2019, 1-12.

[52] S Martin, S Vora, K Yune, et al. Dynamics of driver's gaze explorations in behavior modeling \& maneuver prediction. IEEE Transactions on Vehicles, 2018

[53] Y Zhang, J Li, Y Guo, et al. Vehicle driving behavior recognition based on multi-view convolutional neural network with joint data augmentation. IEEE Transactions on Vehicular Technology, 2019, 68(5): 4223-4234.

[54] H Liu, T Taniguchi, Y Tanaka, et al. Visualization of driving behavior based on hidden feature extraction by using deep learning. IEEE Transactions on Intelligent Transportation Systems, 2017, 18(9): 2477-2489.

[55] RToledo-Moreo, M A Zamora-Izquierdo. Collision avoidance support in roads with lateral and longitudinal maneuver prediction by fusing GPS/IMU and digital maps. Transportation Research Part C: Emerging Technologies, 2010, 18(4): 611-625.
[56] Z Ouyang, I Niu, M Guizani. Improved vehicle steering pattern recognition by using selected sensor data. IEEE Transactions on Mobile Computing, 2018, 17(6): 1383-1396.

[57] S Lee, M Q Khan, M N Husen. Continuous car driving intent detection using structural pattern recognition. IEEE Transactions on Intelligent Transportation Systems, 2020: 1-13.

[58] M K Park, S Y Lee, C K Kwon, et al. Design of pedestrian target selection with funnel map for pedestrian AEB system. IEEE Transactions on Vehicular Technology, 2016, 66(5): 3597-3609.

[59] NEChelbi, D Gingras, C Sauvageau. Proposal of a new virtual evaluation approach of preventive safety applications and advanced driver assistance functions - application: AEB system. IET Intelligent Transport Systems, 2018, 12(9): 1148-1156.

[60] K Gillmeier, T Schuetttke, F Diederichs, et al. Combined driver distraction and intention algorithm for maneuver prediction and collision avoidance. IEEE International Conference on Vehicular Electronics and Safety, Madrid, Spain, September 12-14, 2018.

[61] F Diederichs, TSchuttke, D Spath. Driver intention algorithm for pedestrian protection and automated emergency braking systems. 2015 IEEE 18th International Conference on Intelligent Transportation Systems, 2015: 1049-1054.

[62] G Li, S E Li, B Cheng, et al. Estimation of driving style in naturalistic highway traffic using maneuver transition probabilities. Transportation Research Part C: Emerging Technologies, 2017, 74: 113-125.

[63] C Lv, Y Xing, C Lu, et al. Hybrid-learning-based classification and quantitative inference of driver braking intensity of an electrified vehicle. IEEE Transactions on Vehicular Technology, 2018, 67(7): 5718-5729.

[64] W Wang, J Xi, D Zhao. Learning and inferring a driver's braking action in car-following scenarios. IEEE Transactions on Vehicular Technology, 2018, 67(5): 3887-3899.

[65] X Zhao, S Wang, J Ma, et al. Identification of driver's braking intention based on a hybrid model of GHMM and GGAP-RBFNN. Neural Computing and Applications, 2018, 31(S1): 161-174.

[66] W Yang, B Wan, X Qu. A forward collision warning system using driving intention recognition of the front vehicle and V2V communication. IEEE Access, 2020, 8: 11268-11278.

[67] S Jia, F Hui, S Li, et al. Long short-term memory and convolutional neural network for abnormal driving behaviour recognition. IET Intelligent Transport Systems, 2020, 14(5): 306-312.

[68] S Wang, X Zhao, Q Yu, et al. Identification of driver braking intention based on long short-term memory (LSTM) network. IEEE Access, 2020, 8: $180422-180432$.

[69] TTeng, L Bi, Y Liu. EEG-based detection of driver emergency braking intention for brain-controlled vehicles. IEEE Transactions on Intelligent Transportation Systems, 2018, 19(6): 1766-1773.

[70] G Phanomchoeng, R Rajamani. New rollover index for the detection of tripped and untripped rollovers. IEEE Transactions on Industrial Electronics, 2013, 60(10): 4726-4736.

[71] HYu, L Güvenc, Ü Özgüner. Heavy-duty vehicle rollover detection and active roll control. IFAC Proceedings Volumes, 2005, 38(1): 128-133.

[72] L Li, Y Lu, R Wang, et al. A three-dimensional dynamics control framework of vehicle lateral stability and rollover prevention via active braking with MPC. IEEE Transactions on Industrial Electronics, 2017, 64(4): 3389-3401.

[73] Y Yin, S Rakheja, P E Boileau. A roll stability performance measure for off-road vehicles. Journal of Terramechanics, 2016, 64: 58-68.

[74] S Lapapong, A A Brown, K S Swanson, et al. Zero-moment point determination of worst-case manoeuvres leading to vehicle wheel lift. Vehicle System Dynamics, 2012, 50(1): 191-214.

[75] C Larish, D Piyabongkarn, V Tsourapas, et al. A new predictive lateral load transfer ratio for rollover prevention systems. IEEE Transactions on Vehicular Technology, 2013, 62(7): 2928-2936.

[76] M Ghazali, M Durali, H Salarieh. Path-following in model predictive rollover prevention using front steering and braking Vehicle System Dynamics, 2016, 55(1): 121-148.

[77] X Qian, C Wang, W Zhao. Rollover prevention and path following control of integrated steering and braking systems. Proceedings of the Institution of Mechanical Engineers, Part D: Journal of Automobile Engineering, 2020, 234(6): 1644-1659. 
[78] H Li, J Li, Z Su, et al. Research on active obstacle avoidance control strategy for intelligent vehicle based on active safety collaborative control. IEEE Access, 2020, 8:183736-183748.

[79] B C Chen, H Peng. Differential-braking-based rollover prevention for sport utility vehicles with human-in-the-loop evaluations. Vehicle System Dynamics, 2010, 36(4-5): 359-389.

[80] CWang, Z Wang, L Zhang, et al. A vehicle rollover evaluation system based on enabling state and parameter estimation. IEEE Transactions on Industrial Informatics, 2020.

[81] R Rajamani, D Piyabongkarn, VTsourapas, et al. Parameter and state estimation in vehicle roll dynamics. IEEE Transactions on Intelligent Transportation Systems, 2011, 12(4): 1558-1567.

[82] K Nam, S Oh, H Fujimoto, et al. Estimation of sideslip and roll angles of electric vehicles using lateral tire force sensors through RLS and Kalman filter approaches. IEEE Transactions on Industrial Electronics, 2013, 60(3): 988-1000.

[83] Z Ma, X Ji, Y Zhang, et al. State estimation in roll dynamics for commercial vehicles. Vehicle System Dynamics, 2016, 55(3): 313-337.

[84] J G Guzman, L P Gonzalez, J P Redondo, et al. Real-time vehicle roll angle estimation based on neural networks in loT low-cost devices. Sensors (Basel), 2018, 18(7).

[85] H Imine, A Benallegue, T Madani, et al. Rollover risk prediction of heavy vehicle using high-order sliding-mode observer: Experimental results. IEEE Transactions on Vehicular Technology, 2014, 63(6): 2533-2543.

[86] D Chu, Z Li, J Wang, et al. Rollover speed prediction on curves for heavy vehicles using mobile smartphone. Measurement, 2018, 130: 404-411.

[87] SYim, J Choi, KYi. Coordinated control of hybrid 4 WD vehicles for enhanced maneuverability and lateral stability. IEEE Transactions on Vehicular Technology, 2012, 61(4): 1946-1950.

[88] A Tahouni, M Mirzaei, B Najjari. Novel constrained nonlinear control of vehicle dynamics using integrated active torque vectoring and electronic stability control. IEEE Transactions on Vehicular Technology, 2019, 68(10): 9564-9572.

[89] Z Wang, J Zhu, L Zhang, et al. Automotive ABS/DYC coordinated control under complex driving conditions. IEEE Access, 2018, 6: 32769-32779.

[90] M Nagai, Y Hirano, S Yamanaka. Integrated robust control of active rear wheel steering and direct yaw moment control. Vehicle System Dynamics, 1998, 29(1): 416-421.

[91] E Mousavinejad, Q L Han, F Yang, et al. Integrated control of ground vehicles dynamics via advanced terminal sliding mode control. Vehicle System Dynamics, 2016, 55(2): 268-294.

[92] J Hu, Z Hu, C Fu, et al. Integrated control of AFS and DYC for in-wheelmotor electric vehicles based on operation region division. International Journal of Vehicle Design, 2019, 79(4): 221-247.

[93] J Feng, S Chen, Z Qi. Coordinated chassis control of 4WD vehicles utilizing differential braking, traction distribution and active front steering. IEEE Access, 2020, 8: 81055-81068.

[94] M Jalali, S Khosravani, A Khajepour, et al. Model predictive control of vehicle stability using coordinated active steering and differential brakes. Mechatronics, 2017, 48: 30-41.

[95] S D Cairano, H E Tseng, D Bernardini, et al. Vehicle yaw stability control by coordinated active front steering and differential braking in the tire sideslip angles domain. IEEE Transactions on Control Systems Technology, 2013, 21(4): 1236-1248.

[96] M Choi, S B Choi. MPC for vehicle lateral stability via differential braking and active front steering considering practical aspects. Proceedings of the Institution of Mechanical Engineers, Part D: Journal of Automobile Engineering, 2015, 230(4): 459-469.

[97] X Huang, $\mathrm{H}$ Zhang, G Zhang, et al. Robust weighted gain-scheduling $\mathrm{H}$ vehicle lateral motion control with considerations of steering system Backlash-type hysteresis. IEEE Transactions on Control Systems Technology, 2014, 22(5): 1740-1753.

[98] X Yang, Z Wang, W Peng. Coordinated control of AFS and DYC for vehicle handling and stability based on optimal guaranteed cost theory. Vehicle System Dynamics, 2009, 47(1): 57-79.

[99] Y Hao, V Cocquempot, J Bin. Optimal fault-tolerant path-tracking control for 4WS4WD electric vehicles. IEEE Transactions on Intelligent Transportation Systems, 2010, 11(1): 237-243.

[100] W Cho, J Choi, C Kim, et al. Unified chassis control for the improvement of agility, maneuverability, and lateral stability. IEEE Transactions on Vehicular Technology, 2012, 61(3): 1008-1020.
[101] B A Güvenç, L Güvenç, T Yiğit, et al. Coordination strategies for combined steering and individual wheel braking actuated vehicle yaw stability control. IFAC Proceedings Volumes, 2004, 37(22): 85-90.

[102] M Doumiati, O Sename, L Dugard, et al. Integrated vehicle dynamics control via coordination of active front steering and rear braking. European Journal of Control, 2013, 19(2): 121-143.

[103] N Ding, S Taheri. An adaptive integrated algorithm for active front steering and direct yaw moment control based on direct Lyapunov method. Vehicle System Dynamics, 2010, 48(10): 1193-1213.

[104] M Nagai. The perspectives of research for enhancing active safety based on advanced control technology. Vehicle System Dynamics, 2007, 45(5): 413-431.

[105] S Ç Bașlamișli, I E Köse, G Anlaç. Handling stability improvement through robust active front steering and active differential control. Vehicle System Dynamics, 2010, 49(5): 657-683.

[106] J Ni, J Hu, C Xiang. Envelope control for four-wheel independently actuated autonomous ground vehicle through AFS/DYC integrated control. IEEE Transactions on Vehicular Technology, 2017, 66(11): 9712-9726.

[107] Q Meng, T Zhao, C Qian, et al. Integrated stability control of AFS and DYC for electric vehicle based on non-smooth control. International Journal of Systems Science, 2018, 49(7): 1518-1528.

[108] X Jin, Z Yu, G Yin, et al. Improving vehicle handling stability based on combined AFS and DYC system via robust Takagi-Sugeno fuzzy control. IEEE Transactions on Intelligent Transportation Systems, 2018, 19(8): 2696-2707.

[109] M Mirzaei, H Mirzaeinejad. Fuzzy scheduled optimal control of integrated vehicle braking and steering systems. IEEE/ASME Transactions on Mechatronics, 2017, 22(5): 2369-2379.

[110] S Ç Baslamisli, I E Köse, G Anlaş. Gain-scheduled integrated active steering and differential control for vehicle handling improvement. Vehicle System Dynamics, 2009, 47(1): 99-119.

[111] L Li, FY Wang, Q Zhou. Integrated longitudinal and lateral tire/road friction modeling and monitoring for vehicle motion control. IEEE Transactions on Intelligent Transportation Systems, 2006, 7(1): 1-19.

[112] P Song, M Tomizuka, C Zong. A novel integrated chassis controller for full drive-by-wire vehicles. Vehicle System Dynamics, 2015, 53(2): 215-236.

[113] O Mokhiamar, M Abe. Active wheel steering and yaw moment control combination to maximize stability as well as vehicle responsiveness during quick lane change for active vehicle handling safety. Proceedings of the Institution of Mechanical Engineers, Part D: Journal of Automobile Engineering, 2005, 216(2): 115-124

[114] TWeiskircher, S Müller. Control performance of a road vehicle with four independent single-wheel electric motors and steer-by-wire system. Vehicle System Dynamics, 2012, 50(1): 53-69.

[115] J Zhang, W Sun, H Du. Integrated motion control scheme for fourwheel-independent vehicles considering critical conditions. IEEE Transactions on Vehicular Technology, 2019, 68(8): 7488-7497.

[116] E Ono, Y Hattori, Y Muragishi, et al. Vehicle dynamics integrated control for four-wheel-distributed steering and four-wheel-distributed traction/ braking systems. Vehicle System Dynamics, 2006, 44(2): 139-151.

[117] J Ahmadi, A K Sedigh, M Kabganian. Adaptive vehicle lateral-plane motion control using optimal tire friction forces with saturation limits consideration. IEEE Transactions on Vehicular Technology, 2009, 58(8): 4098-4107.

[118] J He, D A Crolla, M C Levesley, et al. Coordination of active steering, driveline, and braking for integrated vehicle dynamics control. Proceedings of the Institution of Mechanical Engineers, Part D: Journal of Automobile Engineering, 2006, 220(10): 1401-1420.

[119] J XWang, N Chen, D W Pi, et al. Agent-based coordination framework for integrated vehicle chassis control. Proceedings of the Institution of Mechanical Engineers, Part D: Journal of Automobile Engineering, 2009, 223(5): 601-621.

[120] F Fahimi. Full drive-by-wire dynamic control for four-wheel-steer allwheel-drive vehicles. Vehicle System Dynamics, 2013, 51 (3): 360-376.

[121] J Andreasson, T Bünte. Global chassis control based on inverse vehicle dynamics models. Vehicle System Dynamics, 2006, 44(1): 321-328.

[122] C Chen, Y Jia, M Shu, et al. Hierarchical adaptive path-tracking control for autonomous vehicles. IEEE Transactions on Intelligent Transportation Systems, 2015, 16(5): 2900-2912. 
[123] P Falcone, H ETseng, F Borrelli, et al. MPC-based yaw and lateral stabilisation via active front steering and braking. Vehicle System Dynamics, 2008, 46(1): 611-628.

[124] H Peng, W Wang, C Xiang, et al. Torque coordinated control of four in-wheel motor independent-drive vehicles with consideration of the safety and economy. IEEE Transactions on Vehicular Technology, 2019, 68(10): 9604-9618.

[125] J Zhu, Z Wang, L Zhang, et al. Braking/steering coordination control for in-wheel motor drive electric vehicles based on nonlinear model predictive control. Mechanism and Machine Theory, 2019, 142.

[126] J Guo, P Hu, R Wang. Nonlinear coordinated steering and braking control of vision-based autonomous vehicles in emergency obstacle avoidance. IEEE Transactions on Intelligent Transportation Systems, 2016, 17(11): 3230-3240

[127] V Fors, B Olofsson, L Nielsen. Formulation and interpretation of optimal braking and steering patterns towards autonomous safety-critical manoeuvres. Vehicle System Dynamics, 2018, 57(8): 1206-1223.

[128] A S P Singh, O Nishihara. Nondimensionalized univariate equation characterizing optimal state feedback control for collision avoidance. IEEE Transactions on Intelligent Transportation Systems, 2018, 19(10): 3344-3359.

[129] Q Cui, R Ding, X Wu, et al. A new strategy for rear-end collision avoidance via autonomous steering and differential braking in highway driving. Vehicle System Dynamics, 2019: 1-32.

[130] FWang, H Chen, D Cao. Nonlinear coordinated motion control of road vehicles after a tire blowout. IEEE Transactions on Control Systems Technology, 2016, 24(3): 956-970.

[131] R Hajiloo, M Abroshan, A Khajepour, et al. Integrated steering and differential braking for emergency collision avoidance in autonomous vehicles. IEEE Transactions on Intelligent Transportation Systems, 2020: 1-12.

[132] [132]J Funke, M Brown, S M Erlien, et al. Collision avoidance and stabilization for autonomous vehicles in emergency scenarios. IEEE Transactions on Control Systems Technology, 2017, 25(4): 1204-1216.

[133] S Cheng, L Li, H Guo, et al. Longitudinal collision avoidance and lateral stability adaptive control system based on MPC of autonomous vehicles. IEEE Transactions on Intelligent Transportation Systems, 2019: 1-10.

[134] J Kang, J Yoo, K Yi. Driving control algorithm for maneuverability, lateral stability, and rollover prevention of 4WD electric vehicles with independently driven front and rear wheels. IEEE Transactions on Vehicular Technology, 2011, 60(7): 2987-3001.

[135] H Imine, L M Fridman, T Madani. Steering control for rollover avoidance of heavy vehicles. IEEE Transactions on Vehicular Technology, 2012, 61 (8): 3499-3509.

[136] R Rajamani, D N Piyabongkarn. New paradigms for the integration of yaw stability and rollover prevention functions in vehicle stability control. IEEE Transactions on Intelligent Transportation Systems, 2013, 14(1): 249-261.

[137] M Cao, G Wu, S Yan, et al. Control strategy of vehicle anti-rollover considering driver's characteristic. IEEE Access, 2020, 8: 128264-128281.

[138] H Her, Y Koh, E Joa, et al. An integrated control of differential braking, front/rear traction, and active roll moment for limit handling performance. IEEE Transactions on Vehicular Technology, 2016, 65(6): 4288-4300.

[139] C Spelta, F Previdi, P Bolzern, et al. Performance analysis of semi-active suspensions with control of variable damping and stiffness. Vehicle System Dynamics, 2011, 49(1-2): 237-256.

[140] H Habibi, K H Shirazi, M Shishesaz. Roll steer minimization of McPherson-strut suspension system using genetic algorithm method. Mechanism and Machine Theory, 2008, 43(1): 57-67.

[141] Y Li, W Sun, J Huang, et al. Effect of vertical and lateral coupling between tyre and road on vehicle rollover. Vehicle System Dynamics, 2013, 51(8): 1216-1241.

[142] B Nemeth, D Fenyes, P Gaspar, et al. Coordination of independent steering and torque vectoring in a variable-geometry suspension system. IEEE Transactions on Control Systems Technology, 2019, 27(5): 2209-2220.

[143] M Akar, J C Kalkkuhl. Design and evaluation of an integrated chassis controller for automotive vehicle emulation. IEEE Transactions on Industrial Electronics, 2009, 56(9): 3571-3579.

[144] C March, T Shim. Integrated control of suspension and front steering to enhance vehicle handling. Proceedings of the Institution of Mechanical
Engineers, Part D: Journal of Automobile Engineering, 2007, 221(4): 377-391.

[145] S Bei, L Chen, B Chen, et al. On fuzzy-PID integrated control of automotive electric power steering and semi-active suspension. 2008 Second International Symposium on Intelligent Information Technology Application, 2008: 847-851.

[146] H Imine, M Djemai. Switched control for reducing impact of vertical forces on road and heavy-vehicle rollover avoidance. IEEE Transactions on Vehicular Technology, 2016, 65(6): 4044-4052.

[147] H Du, N Zhang, L Wang. Switched control of vehicle suspension based on motion-mode detection. Vehicle System Dynamics, 2013, 52(1): 142-165.

[148] M Akar, A D Dere. A switching rollover controller coupled with closedloop adaptive vehicle parameter identification. IEEE Transactions on Intelligent Transportation Systems, 2014, 15(4): 1579-1585.

[149] H Dahmani, O Pages, A E Hajjaji. Observer-based state feedback control for vehicle chassis stability in critical situations. IEEE Transactions on Control Systems Technology, 2015.

[150] X Jin, G Yin, C Bian, et al. Gain-scheduled vehicle handling stability control via integration of active front steering and suspension systems. Journal of Dynamic Systems, Measurement, and Control, 2016, 138(1).

[151] H A Hamersma, P S Els. Improving the braking performance of a vehicle with $A B S$ and a semi-active suspension system on a rough road. Journal of Terramechanics, 2014, 56: 91-101.

[152] W Wang, M Chen, S Su. Hierarchical fuzzy-neural control of anti-lock braking system and active suspension in a vehicle. Automatica, 2012, 48(8): 1698-1706.

[153] J Edrén, M Jonasson, J Jerrelind, et al. Utilisation of optimisation solutions to control active suspension for decreased braking distance. Vehicle System Dynamics, 2015, 53(2): 256-273.

[154] J S Lin, W E Ting. Nonlinear control design of anti-lock braking systems with assistance of active suspension. IET Control Theory \& Applications, 2007, 1(1): 343-348.

[155] J Zhang, W Sun, H Jing. Nonlinear robust control of antilock braking systems assisted by active suspensions for automobile. IEEE Transactions on Control Systems Technology, 2019, 27(3): 1352-1359.

[156] W Wang, Y Chien, M Chen, et al. Control of uncertain active suspension system with antilock braking system using fuzzy neural controllers. Proceedings of the 2009 IEEE International Conference on Systems, Man, and Cybernetics, San Antonio, TX, USA, October, 2009, 3371-3376.

[157] Z Wu, Y Liu, G Pan. A smart car control model for brake comfort based on car following. IEEE Transactions on Intelligent Transportation Systems, 2009, 10(1): 42-46.

[158] H Xiao, W Chen, $\mathrm{H}$ Zhou, et al. Integrated control of active suspension system and electronic stability programme using hierarchical control strategy: theory and experiment. Vehicle System Dynamics, 2011, 49(12): 381-397.

[159] R Tchamna, E Youn, I Youn. Combined control effects of brake and active suspension control on the global safety of a full-car nonlinear model. Vehicle System Dynamics, 2014, 52(1): 69-91.

[160] C Chu, W Chen. Integrated design of vehicle suspension and braking systems based on layered coordinated control strategy. SAE Technical Paper Series, Detroit, Michigan, April 14-17, 2008.

[161] J Zhang, W Sun, M Zeng. Active suspension switching control from antilock braking assistance to ride comfort improvement. Proceedings of the 37th Chinese Control Conference, Wuhan, China, July 25-27, 2018.

[162] C P Vassal, O Sename, L Dugard, et al. Attitude and handling improvements through gain-scheduled suspensions and brakes control. Control Engineering Practice, 2011, 19(3): 252-263.

[163] H Chou, B D Novel. Global vehicle control using differential braking torques and active suspension forces. Vehicle System Dynamics, 2005, 43(4): 261-284.

[164] Y Jangyeol, C Wanki, K Bongyeong, et al. Unified chassis control for rollover prevention and lateral stability. IEEE Transactions on Vehicular Technology, 2009, 58(2): 596-609.

[165] Y Furukawa, M Abe. Advanced chassis control systems for vehicle handling and active safety. Vehicle System Dynamics, 1997, 28(2-3): 59-86.

[166] L Zhang, Y Wang, Z Wang. Robust lateral motion control for in-wheelmotor-drive electric vehicles with network induced delays. IEEE Transactions on Vehicular Technology, 2019, 68(11): 10585-10593. 
[167] X Ding, Z Wang, L Zhang, et al. Longitudinal vehicle speed estimation for four-wheel-independently-actuated electric vehicles based on multi-sensor fusion. IEEE Transactions on Vehicular Technology, 2020, 69(11): 12797-12806.

[168] H Zhao, W Chen, J Zhao, et al. Modular integrated longitudinal, lateral, and vertical vehicle stability control for distributed electric vehicles. IEEE Transactions on Vehicular Technology, 2019, 68(2): 1327-1338.

[169] D Li, S Du, F Yu. Integrated vehicle chassis control based on direct yaw moment, active steering and active stabiliser. Vehicle System Dynamics, 2008, 46(1): 341-351.

[170] W Cho, J Yoon, J Kim, et al. An investigation into unified chassis control scheme for optimised vehicle stability and manoeuvrability. Vehicle System Dynamics, 2008, 46(1): 87-105.

[171] S B Lu, S B Choi, Y N Li, et al. Global integrated control of vehicle suspension and chassis key subsystems. Proceedings of the Institution of Mechanical Engineers, Part D: Journal of Automobile Engineering, 2010, 224(4): 423-441

[172] H Termous, H Shraim, R Talj, et al. Coordinated control strategies for active steering, differential braking and active suspension for vehicle stability, handling and safety improvement. Vehicle System Dynamics, 2018, 57(10): 1494-1529.

[173] Z He, X Ji. Nonlinear robust control of integrated vehicle dynamics. Vehicle System Dynamics, 2012, 50(2): 247-280.
[174] T Gordon, M Howell, F Brandao. Integrated control methodologies for road vehicles. Vehicle System Dynamics, 2003, 40(1-3): 157-190.

[175] J Zhao, P K Wong, X Ma, et al. Chassis integrated control for active suspension, active front steering and direct yaw moment systems using hierarchical strategy. Vehicle System Dynamics, 2016, 55(1): 72-103.

[176] M Čorić, J Deur, L Xu, et al. Optimisation of active suspension control inputs for improved performance of active safety systems. Vehicle System Dynamics, 2017, 56(1): 1-26.

[177] H Her, J Suh, KYi. Integrated control of the differential braking, the suspension damping force and the active roll moment for improvement in the agility and the stability. Proceedings of the Institution of Mechanical Engineers, Part D: Journal of Automobile Engineering, 2014, 229(9): 1145-1157.

[178] S Fergani, O Sename, L Dugard. An integrated vehicle dynamic controller. IEEE Transactions on Vehicular Technology, 2016, 65(4): 1880-1889.

[179] S B Lu, Y N Li, S B Choi. Contribution of chassis key subsystems to rollover stability control. Proceedings of the Institution of Mechanical Engineers, Part D: Journal of Automobile Engineering, 2011, 226(4): 479-493.

[180] W Li, Z Xie, P K Wong, et al. Adaptive-event-trigger-based fuzzy nonlinear lateral dynamic control for autonomous electric vehicles under insecure communication networks. IEEE Transactions on Industrial Electronics, 2021, 68(3): 2447-2459.

\section{Submit your manuscript to a SpringerOpen ${ }^{\circ}$ journal and benefit from:}

- Convenient online submission

- Rigorous peer review

- Open access: articles freely available online

- High visibility within the field

- Retaining the copyright to your article

Submit your next manuscript at $\boldsymbol{\nabla}$ springeropen.com 